\title{
Top-quark pair production at hadron colliders: differential cross section and phenomenological applications with DiffTop
}

\author{
Marco Guzzi, ${ }^{a}$ Katerina Lipka ${ }^{a}$ and Sven-Olaf Moch $^{b, c}$ \\ ${ }^{a}$ Deutsches Elektronen-Synchrotron DESY, \\ Notkestrasse 85, D-22607 Hamburg, Germany \\ ${ }^{b}$ II. Institut für Theoretische Physik, Universität Hamburg, \\ Luruper Chaussee 149, D-22761 Hamburg, Germany \\ ${ }^{c}$ Deutsches Elektronen-Synchrotron DESY, \\ Platanenallee 6, D-15738 Zeuthen, Germany \\ E-mail: marco.guzzi@desy.de, katerina.lipka@desy.de, \\ sven-olaf.moch@desy.de
}

ABSTRACT: The results of phenomenological studies of top-quark pair production in proton-proton collisions are presented. Differential cross sections are calculated in perturbative QCD at approximate next-to-next-to-leading order $\mathcal{O}\left(\alpha_{s}^{4}\right)$ by using methods of threshold resummation beyond the leading logarithmic accuracy. Predictions for the singleparticle inclusive kinematics are presented for transverse momentum and rapidity distributions of final-state top quarks. Uncertainties related to the description of proton structure, top-quark mass and strong coupling constant are investigated in detail. The results are compared to the recent measurements by the ATLAS and CMS collaborations at the LHC at the center of mass energy of $7 \mathrm{TeV}$. The calculation presented here is implemented in the computer code DiffTop and can be applied to the general case of heavy-quark pair production at hadron-hadron colliders. For the first time, a fit of parton distribution functions at NNLO is performed by using the differential cross sections of top-quark pair production together with other data sets. The impact of the top-pair production on the precision of the gluon distribution at high scales is illustrated.

KEywords: QCD Phenomenology, Hadronic Colliders

ARXIV EPRINT: 1406.0386 


\section{Contents}

1 Introduction 1

1.1 Probing the proton structure through $t \bar{t}$ production 2

1.2 Threshold resummation techniques 3

2 Single particle kinematic $\quad 5$

$\begin{array}{lll}3 & \text { Overview of the threshold resummation calculation } & 7\end{array}$

4 Sensitivity of $t \bar{t}$ production to PDF-related aspects of QCD 10

4.1 Cross sections of $t \bar{t}$ production at approximate NNLO obtained with different PDFs

4.2 QCD analysis using the $t \bar{t}$ production measurements

$\begin{array}{lll}5 & \text { Summary and conclusions } & 17\end{array}$

$\begin{array}{ll}\text { A Comparison with other PDF sets } & 19\end{array}$

\section{Introduction}

Studies of heavy-quark production at hadron colliders provide stringent tests of quantum chromodynamics (QCD) and of the theory of electroweak (EW) interactions. Furthermore, these are of crucial importance in searches for signatures of physics Beyond the Standard Model (BSM). The mass of the recently discovered Higgs boson [1, 2] has been measured to be in the range $120 \leq m_{H} \leq 135 \mathrm{GeV}$, therefore the Higgs sector is expected to be closely related to the physics of the top-quark. In particular, the role of quantum corrections to the top-quark mass, which together with the mass of the Higgs-boson define the electroweak vacuum stability conditions has been studied $[3,4]$.

Experimentally, top-quark physics is being studied at the Tevatron and is extensively explored at the unprecedented energies of the Large Hadron Collider (LHC). The CMS, ATLAS, DØ, and CDF collaborations have recently combined their results [5] for the topquark mass and obtained a value of $m_{t}=173.3 \pm 0.76 \mathrm{GeV}$. The interpretation of the measured mass and its relation to the theoretically well-defined pole mass of the top quark is discussed in details in [6]. New observables to be used for the measurement of the topquark mass at hadron colliders are proposed in [7, 8]. The most recent determination of the pole mass of the top quark is performed by the CMS collaboration [9]. In the same analysis, for the first time, the issue of correlations between the top-quark pole mass, gluon distribution, and strong coupling constant $\alpha_{s}$ in QCD predictions for the inclusive cross section of top-quark pair production is discussed. 
Precise measurements for the total and differential cross section for top-quark pair production at a center-of-mass energies $\sqrt{S}=7$ and $8 \mathrm{TeV}$ have been recently published by the CMS $[10,11]$ and ATLAS [12-15] collaborations. The interpretation of current and forthcoming LHC data demands high-precision theory predictions that imply a new realm of precision calculations in perturbative QCD (pQCD), supplied by the development of efficient tools for phenomenological analyses. The QCD corrections to heavy-quark production at hadron colliders at the next-to-leading order (NLO), $\mathcal{O}\left(\alpha_{s}^{3}\right)$, are known since many years [16-21]. The full calculation at next-to-next-to-leading order (NNLO), $\mathcal{O}\left(\alpha_{s}^{4}\right)$, for the inclusive cross section has been accomplished only recently [22-25] and required continuous efforts of the QCD community in calculating radiative corrections and in the development of computational tools [26-33]. The NNLO calculation of the inclusive cross section for the $t \bar{t}$ production is implemented in the $\mathrm{C}++$ computer programs Top $++[34]$ and Hathor [35]. In these calculations the final-state top quarks are considered in the on-shell approximation. Studies in QCD at NLO where final-state top quarks decay into pairs of $W$ bosons and $b$ quarks can be found in refs. [36-38].

The comparison of QCD predictions with the data and a multitude of phenomenological analyses of interest require to have precise predictions not only for the inclusive cross section, but also at differential level. Invariant mass distribution of $t \bar{t}$, transverse momentum and rapidity distributions of the top quark or $t \bar{t}$, are examples of differential distributions needed at the highest perturbative order possible. The exact NLO calculations for $t \bar{t}$ total and differential cross sections are implemented into Monte Carlo (MC) numerical codes MCFM [39], MC@NLO [40], POWHEG [41], MadGraph/MadEvent [42, 43]. The NNLO corrections for these observables are not yet available.

In this paper, we present the results of a phenomenological analysis of differential cross section of $t \bar{t}$ production at the LHC, in which the estimate of the uncertainties due to the knowledge of the proton structure is addressed. The recent inclusive and, for the first time, differential measurements of $t \bar{t}$ production at hadron colliders are included in a QCD analysis at NNLO. The impact of such data on the uncertainty of the gluon distribution is illustrated. For this purpose, the necessary developments of computing tools based on the available theory had to be performed. The approximate NNLO $\mathcal{O}\left(\alpha_{s}^{4}\right)$ calculation for the differential cross section in the single-particle inclusive (1PI) kinematic for heavy-flavor production at hadron colliders has been implemented in a novel computer code DifFToP which we provide at [44]. In this calculation, techniques of logarithmic expansion beyond the leading logarithmic accuracy in QCD threshold resummation are used.

\subsection{Probing the proton structure through $t \bar{t}$ production}

Top-pair production at the LHC probes parton distribution functions (PDFs) of the proton, in particular the gluon distribution. In proton-proton collisions at the LHC, $t \bar{t}$ production is mainly driven by the gluon, in fact approximately $85 \%$ of the total cross section is ascribed to the gluon-gluon channel at $\sqrt{S}=7 \mathrm{TeV}$ and this fraction grows with the increase of $\sqrt{S}$. Measurements of the $t \bar{t}$ total and differential cross sections offer the possibility of probing the gluon in the large Bjorken $x$ region $(x \approx 0.1)$ where gluon is currently poorly constrained. This has first been studied in [45] (see also [46]). However, strong correlations 
between $\alpha_{s}$, gluon distribution $g(x)$, and the pole mass of the top quark $m_{t}$, in the QCD description of $t \bar{t}$ production have to be taken into account. A simultaneous determination of $g(x), \alpha_{s}$ and $m_{t}$, using the $t \bar{t}$ measurements at the LHC in a QCD analysis, together with relevant measurements in Deep-Inelastic Scattering (DIS) and in proton-(anti)proton collisions might resolve these correlations.

The developed program and computational tools used for the phenomenological analyses presented in this paper, provide a basis for the inclusion of differential $t \bar{t}$ cross sections into QCD analyses at NNLO. For the purpose of a fast calculation within QCD analyses for PDF determination, DifFTop is interfaced to FASTNLO [47-50] and included into the QCD analysis platform HERAFitTER [51]. This allows the user to perform, for the first time, full genuine PDF fits including differential cross sections of $t \bar{t}$ production by using fast theoretical predictions for these observables. This not only represents a clear advantage in terms of the CPU-time consumption, but also opens the window to fully explore the potential of the $t \bar{t}$ measurements. The exact NNLO calculation for the fully differential $t \bar{t}$ distributions, once available, will be of clear advantage. However, full global QCD analyses of the current and forthcoming high-precision LHC measurements set specific requirements to the representation of the experimental data and availability of fast computing tools. The present analysis addresses these requirements in the context of differential $t \bar{t}$ production cross sections for the first time, by using approximate calculations in order to facilitate future PDF fits using the exact NNLO theory.

Fast theoretical predictions for the full NNLO differential cross section calculation can be in principle obtained by using tools like FASTNLO [47-50] or APPLGRID [52]. The grid files generated in the output of such codes can be directly read inside the HERAFitTER open-source platform to determine the impact of the experimental $t \bar{t}$ data on PDFs by performing the QCD analysis. Presumably, the full NNLO calculation will be lengthy and CPU time consuming and the complication for creating an interface to FASTNLO or APPLGRID will depend upon the structure of the calculation itself and the way the integration is done. One can forsee that the generation of FASTNLO/APPLGRID grids will be feasible, but it might happen that it is dramatically CPU time consuming. If that will be the case, a possible way out will be to work on approximate calculations which can be highly improved once the full calculation is available.

\subsection{Threshold resummation techniques}

The new LHC measurements give us the possibility of investigating the applicability of QCD factorization for hadronic cross sections with high precision. In the past three decades QCD techniques have experienced stages of remarkable progress on theoretical and phenomenological ground. In particular, theoretical tools were introduced to estimate the importance of the perturbative higher orders in cross-section calculations [53-58]. In figure 1 we show the top-quark transverse momentum, $p_{T}^{t}$, where higher orders are important and have reduced scale dependence.

The non-unique separation between long- and short-distance dynamics of partons in the proton is understood in terms of factorization theorems, in which inclusive hadronic cross sections are factorized in universal nonperturbative PDFs and fragmentation func- 

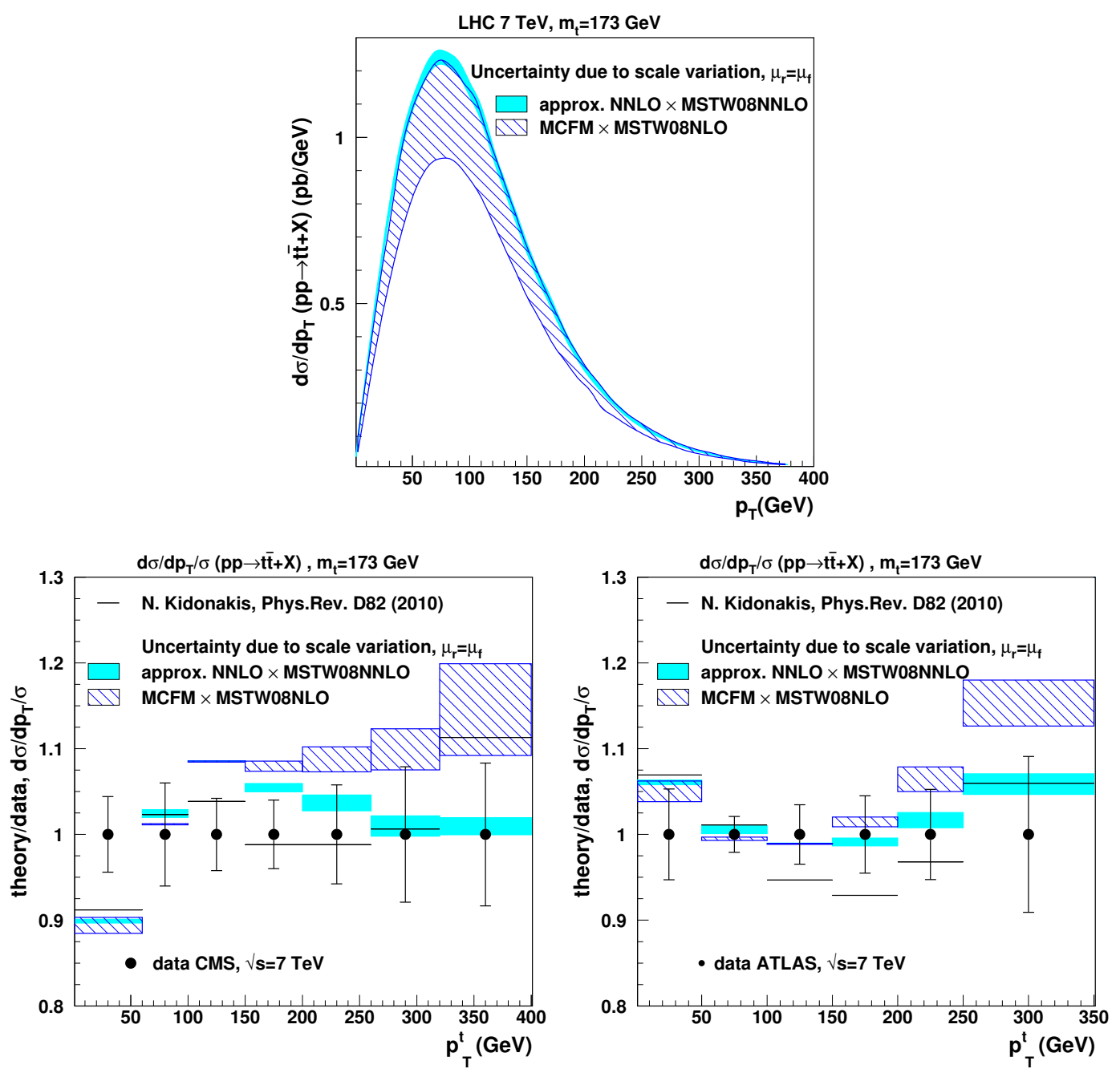

Figure 1. Study of scale uncertainties for MCFM and DifFTop calculations for the top-quark $p_{T}^{t}$ distribution. Upper inset: absolute $p_{T}^{t}$ distribution at NLO with MCFM (hatched band), and approx. NNLO with DiffTop (shaded band). Lower inset: ratio of theory over data for CMS (left) [10] and ATLAS (right) [12] measurements. Here MSTW08 NLO (NNLO) PDFs are used for the MCFM (DifFTop) calculation. Renormalization and factorization scales are set to $\mu_{R}=\mu_{F}=m_{t}$ and varied such as $m_{t} / 2 \leq \mu_{R}=\mu_{F} \leq 2 m_{t}$. For comparison, the central prediction by N. Kidonakis (solid line) using MSTW08 NNLO PDFs is shown.

tions (FF), and hard scattering coefficients that can be computed in perturbative QCD. These hard scattering functions still contain, in certain kinematic regions, residuals of longdistance dynamic related to leftovers in the cancellation between real and virtual soft-gluon contributions. These finite reminders limit the predictive power of QCD in the kinematic region close to the partonic production threshold where singular logarithmic terms give large corrections to the cross section. Threshold resummation methods, which are based on the universality of the factorization in the soft and collinear limit, allow us to resum these large contributions to all orders so that the predictive power of pQCD is extended to the phase space regions of the partonic threshold. By using threshold resummation methods 
one can derive approximate formulas at NNLO for differential distributions, in which the cross sections are expanded in terms of the logarithmic enhanced contributions (appearing as plus distributions), and can therefore be written at various degrees of logarithmic accuracy. Studies at the next-to-leading-logarithmic (NLL), next-to-next-to-leading-logarithmic (NNLL) accuracy and beyond can be found in refs. [59-68] and references therein.

Recently, methods of heavy-quark effective theory (HQET) and soft-collinear effective theory (SCET) experienced enormous progress and received significant attention. Such methods can be also used to write the factorization formula for the hard-scattering kernels in the threshold region [69-72]. Approximate NNLO calculations, based on SCET, for differential cross sections for heavy-quark production can be found in [73-79] and references therein. A study of differences and similarities between the traditional QCD threshold resummation formalism and SCET can be found in [80] for the $t \bar{t}$ case.

\section{Single particle kinematic}

Top-quark pairs are produced in hadronic reactions in which the scattering process is defined by

$$
H_{1}\left(P_{1}\right)+H_{2}\left(P_{2}\right) \rightarrow t\left(p_{1}\right)+\bar{t}\left(p_{2}\right)+X(k)
$$

where $H_{1}$ and $H_{2}$ are the incoming hadrons with momenta $P_{1}$ and $P_{2}$ respectively, the final state top-quark has momentum $p_{1}$, the anti-top momentum $p_{2}$ and $X(k)$ represents any inclusive hadronic final state allowed by the reaction. At phenomenological level, the differential cross sections of interest are written in such a way that can be compared to measurements that are related to the detection of single particles or particle pairs.

When a single particle is detected in the final state, for instance the top-quark, the oneparticle inclusive (1PI) kinematic is used to determine the $p_{T}^{t}$ spectrum and the rapidity $y^{t}$ distribution of the top-quark. These distributions are obtained by integrating over the phase space of the anti-top (not observed) together with any real emission of radiation [57].

When particle pairs are detected, the pair-invariant mass kinematic (PIM) is used to write a factorized cross section $[53,54,56,58,81]$ in terms of the invariant mass of the heavy system, collective rapidity, and additional variables like the angle between the final-state quark direction and the beam axis. In what follows we will discuss differential distributions derived in the 1PI kinematic case and will leave the PIM kinematic [61] for a forthcoming analysis. ${ }^{1}$

In the case of single particle detection, the reaction in eq. (2.1) is written as

$$
H_{1}\left(P_{1}\right)+H_{2}\left(P_{2}\right) \rightarrow t\left(p_{1}\right)+X[\bar{t}]\left(p_{2}^{\prime}\right),
$$

where $p_{2}^{\prime}$ represents the recoil momentum. The Mandelstam invariants at hadronic level are defined as

$$
S=\left(P_{1}+P_{2}\right)^{2}, \quad T_{1}=\left(P_{2}-p_{1}\right)^{2}-m_{t}^{2}, \quad U_{1}=\left(P_{1}-p_{1}\right)^{2}-m_{t}^{2}, \quad S_{4}=S+T_{1}+U_{1} .
$$

\footnotetext{
${ }^{1}$ The extension of DiffTop to calculate approximate NNLO differential cross section for PIM kinematic is under development.
} 
In the vicinity of the threshold the reaction is dominated by the following partonic subprocesses

$$
\begin{aligned}
& q\left(k_{1}\right)+\bar{q}\left(k_{2}\right) \rightarrow t\left(p_{1}\right)+X[\bar{t}]\left(p_{2}^{\prime}\right), \\
& g\left(k_{1}\right)+g\left(k_{2}\right) \rightarrow t\left(p_{1}\right)+X[\bar{t}]\left(p_{2}^{\prime}\right),
\end{aligned}
$$

where the initial-state parton momenta expressed in terms of momentum fractions $x_{1}, x_{2}$ are $k_{1}=x_{1} P_{1}$ and $k_{2}=x_{2} P_{2}$. The recoil momentum $p_{2}^{\prime}=p_{2}+k$ accounts for momentum $p_{2}$ at the threshold and any additional radiation indicated by momentum $k$.

In the vicinity of the partonic threshold, the hadronic final state $X\left[\bar{t}\left(p_{2}^{\prime}\right)\right] \equiv \bar{t}\left(p_{2}\right)$ and the anti-top carries momentum $p_{2}$. The Mandelstam invariants at parton level are defined as

$$
\begin{aligned}
s & =x_{1} x_{2} S=\left(k_{1}+k_{2}\right)^{2}, & & t_{1}=x_{2} T_{1}=\left(k_{2}-p_{1}\right)^{2}-m_{t}^{2}, \\
u_{1} & =x_{1} U_{1}=\left(k_{1}-p_{1}\right)^{2}-m_{t}^{2}, & & s_{4}=s+t_{1}+u_{1},
\end{aligned}
$$

where the inelasticity of the reaction is accounted for by the invariant $s_{4}=p_{2}^{\prime 2}-m_{t}^{2}$.

The factorized differential cross section is written as

$$
\begin{aligned}
S^{2} \frac{d^{2} \sigma\left(S, T_{1}, U_{1}\right)}{d T_{1} d U_{1}}=\sum_{i, j=q, \bar{q}, g} \int_{x_{1}^{-}}^{1} & \frac{d x_{1}}{x_{1}} \int_{x_{2}^{-}}^{1} \frac{d x_{2}}{x_{2}} f_{i / H_{1}}\left(x_{1}, \mu_{F}^{2}\right) f_{j / H_{2}}\left(x_{2}, \mu_{F}^{2}\right) \\
& \times \omega_{i j}\left(s, t_{1}, u_{1}, m_{t}^{2}, \mu_{F}^{2}, \alpha_{s}\left(\mu_{R}^{2}\right)\right)+\mathcal{O}\left(\Lambda^{2} / m_{t}^{2}\right)
\end{aligned}
$$

where $f_{j / H}\left(x, \mu_{F}^{2}\right)$ is the probability of finding the parton $j$ in hadron $H, \mu_{F}$ and $\mu_{R}$ are the factorization and renormalization scales respectively, and $\omega_{i j}$ is the hard scattering cross section which depends on the kinematic of the reaction. Power suppressed terms $\Lambda^{2} / m_{t}^{2}$ are neglected here. The integration limits in the factorization formula are given by

$$
x_{1}^{-}=-\frac{U_{1}}{S+T_{1}}, \quad x_{2}^{-}=-\frac{x_{1} T_{1}}{x_{1} S+U_{1}} .
$$

The double-differential cross section in eq. (2.6) can be expressed in terms of the transverse momentum $p_{T}^{t}$ of the top quark and its rapidity $y$ by observing that

$$
T_{1}=-\sqrt{s} m_{T} e^{y}, \quad U_{1}=-\sqrt{s} m_{T} e^{-y},
$$

where the transverse mass $m_{T}$ is defined as $m_{T}=\sqrt{p_{T}^{2}+m_{t}^{2}}$.

According to QCD resummation, in the organization of the large logarithms at the threshold of the heavy system, the hard scattering $\omega_{i j}$ functions are expanded in terms of singular functions which are plus-distributions of the type ${ }^{2}$

$$
\left[\frac{\ln ^{l}\left(s_{4} / m_{t}^{2}\right)}{s_{4}}\right]_{+}=\lim _{\Delta \rightarrow 0}\left\{\frac{\ln ^{l}\left(s_{4} / m_{t}^{2}\right)}{s_{4}} \theta\left(s_{4}-\Delta\right)+\frac{1}{l+1} \ln ^{l+1}\left(\frac{\Delta}{m_{t}^{2}}\right) \delta\left(s_{4}\right)\right\},
$$

\footnotetext{
${ }^{2}$ This logarithmic structure refers to the 1PI kinematic. In PIM kinematic one has $\left[\ln ^{l}(1-z) /(1-z)\right]_{+}$, where $z=M^{2} / s$ and $M$ is the invariant mass of the final-state heavy system.
} 
where corrections are denoted as leading-logarithmic (LL) if $l=2 i+1$ at $\mathcal{O}\left(\alpha_{s}^{i+3}\right)$ with $i=$ $0,1, \ldots$, as next-to-leading logarithm (NLL) if $l=2 i$, as next-to-next-to-leading logarithm (NNLL) if $l=2 i-1$, and so on.

Since QCD threshold resummation calculations and resummed formulae for inclusive cross sections for heavy-quark pair production have been extensively discussed in the literature in the past years, we will omit explicit derivations unless necessary and limit ourselves to general definitions. The calculation implemented in DifFTop strictly follows the derivation of ref. [61] and references therein, which we refer to for details. Other particulars of this calculation can be found in $[60,62,64,82]$.

\section{Overview of the threshold resummation calculation}

As described in $[57,61]$ the fully resummed expression for the hard scattering cross section of eq. (2.6) in 1PI kinematic is given by a trace in the color-tensor space of operators in the Mellin $N$-moment space

$$
\begin{aligned}
& \omega_{i j}\left[N, s, t_{1}, u_{1}, m_{t}^{2}, \mu_{R}^{2}, \mu_{F}^{2}, \alpha_{s}\left(\mu_{R}\right)\right]=\operatorname{Tr}\left\{H_{i j}\left(s_{4}, t_{1}, u_{1}, m_{t}^{2}, \mu_{R}^{2}, \mu_{F}^{2}\right)\right. \\
& \quad \times \bar{P} \exp \left[\int_{m_{t}}^{m_{t} / N} \frac{d \mu}{\mu}\left(\Gamma_{S}^{i j}\right)^{\dagger}\left(\alpha_{s}(\mu)\right)\right] S_{i j}\left(s_{4}, t_{1}, u_{1}, m_{t}^{2}, \mu_{R}^{2}, \mu_{F}^{2}\right) \\
& \left.\quad \times P \exp \left[\int_{m_{t}}^{m_{t} / N} \frac{d \mu}{\mu} \Gamma_{S}^{i j}\left(\alpha_{s}(\mu)\right)\right]\right\} \exp \left[E_{i}\left(N_{u}, m_{t}, \mu_{F}, \mu_{R}\right)\right] \exp \left[E_{j}\left(N_{t}, m_{t}, \mu_{F}, \mu_{R}\right)\right] \\
& \quad \times \exp \left\{2 \int_{\mu_{R}}^{m_{t}} \frac{d \mu}{\mu}\left[\gamma_{i}\left(\alpha_{s}(\mu)\right)+\gamma_{j}\left(\alpha_{s}(\mu)\right)\right]\right\},
\end{aligned}
$$

where $N_{u}=N\left(-u_{1} / m_{t}^{2}\right), N_{t}=N\left(-t_{1} / m_{t}^{2}\right)$ are the Mellin moments in 1PI kinematic. The functions $H_{i j}=H_{i j}^{(0)}+\left(\alpha_{s} / \pi\right) H_{i j}^{(1)}+\cdots$ and $S_{i j}=S_{i j}^{(0)}+\left(\alpha_{s} / \pi\right) S_{i j}^{(1)}+\cdots$ are the hard and soft functions respectively. The functions $\Gamma_{S}=\left(\alpha_{s} / \pi\right) \Gamma_{S}^{(1)}+\left(\alpha_{s} / \pi\right)^{2} \Gamma_{S}^{(2)}+\cdots$ are the soft anomalous dimension matrices which are path-ordered in $\mu$. Finally, $\gamma_{i}=$ $\left(\alpha_{s} / \pi\right) \gamma_{i}^{(1)}+\left(\alpha_{s} / \pi\right)^{2} \gamma_{i}^{(2)}+\cdots$ are the anomalous dimensions of the quantum field $i=q, g$. In our calculation, $\Gamma_{S}^{(2)}$ at two-loop for the massive case is given in [83, 84].

The exponentials $\exp E_{i}$ and $\exp E_{j}$ represent resummed expressions for the collinear and soft radiation from incoming and outgoing partons respectively, and are defined as

$$
\begin{aligned}
\exp \left[E_{i}\left(N_{u}, m_{t}, \mu_{F}, \mu_{R}\right)\right]= & \exp \left\{E_{i}\left(N_{u}, 2 k_{i} \cdot \zeta\right)\right\} \\
& \times \exp \left\{-2 \int_{\mu_{R}}^{2 k_{i} \cdot \zeta} \frac{d \mu}{\mu} \gamma_{i}\left(\alpha_{s}(\mu)\right)+2 \int_{\mu_{F}}^{2 k_{i} \cdot \zeta} \frac{d \mu}{\mu} \gamma_{i / i}\left(N_{u}, \alpha_{s}(\mu)\right)\right\},
\end{aligned}
$$

where $\gamma_{i / i}$ are the anomalous dimensions of the operator whose matrix element represents the parton density $f_{i / i}$ in the $\overline{\mathrm{MS}}$ scheme. The vector $\zeta=p_{2} / m_{t}$ is used to define the distance from the threshold in 1PI kinematic: $s_{4} / m_{t}^{2} \approx 2(\zeta \cdot k) / m_{t}$. The first exponential 
in eq. (3.2) is defined as

$$
\begin{aligned}
E_{i}\left(N, 2 k_{i} \cdot \zeta\right)=\int_{0}^{\infty} & d w \frac{\left(1-e^{-N w}\right)}{w} \\
& \times\left\{\int_{w^{2}}^{1} \frac{d \lambda}{\lambda} A_{i}\left[\alpha_{s}\left(\sqrt{\lambda} 2 k_{i} \cdot \zeta\right)\right]+\frac{1}{2} \nu^{i}\left[\alpha_{s}\left(w 2 k_{i} \cdot \zeta\right)\right]\right\},
\end{aligned}
$$

where functions $A_{i}$ and $\nu^{i}$ have perturbative expansion in $\alpha_{s}$ whose explicit expressions up to $\mathcal{O}\left(\alpha_{s}^{2}\right)$ can be found in $[61,82]$ for the Feynman and axial gauge.

After an $\alpha_{s}$-expansion of the resummed hard-scattering cross section, the general structure of the double-differential cross section at parton level is given by

$$
\begin{aligned}
s^{2} \frac{d \sigma_{i j}\left(s, t_{1}, u_{1}, \mu_{R}, \mu_{F}\right)}{d t_{1} d u_{1}}= & \omega_{i j}^{(0)}\left(s_{4}, s, t_{1}, u_{1}\right)+\frac{\alpha_{s}}{\pi} \omega_{i j}^{(1)}\left(s_{4}, s, t_{1}, u_{1}\right) \\
& +\left(\frac{\alpha_{s}}{\pi}\right)^{2} \omega_{i j}^{(2)}\left(s_{4}, s, t_{1}, u_{1}\right)
\end{aligned}
$$

where $\omega_{i j}^{(0)}$ is the cross section at the Born level and the one-loop soft-gluon correction beyond the LL approximation can be written as

$$
\begin{aligned}
\omega_{i j}^{(1)}\left(s_{4}, s, t_{1}, u_{1}\right)= & C_{1}^{(1)}\left[\frac{\ln \left(s_{4} / m_{t}^{2}\right)}{s_{4}}\right]_{+}+\left(C_{0}^{(1)}+C_{0, \mu_{F}}^{(1)}\right) \ln \left(\frac{\mu_{F}^{2}}{m_{t}^{2}}\right)\left[\frac{1}{s_{4}}\right]_{+} \\
& +\left[R_{1}+r_{1, \mu_{F}} \ln \left(\frac{\mu_{F}^{2}}{m_{t}^{2}}\right)+r_{1, \mu_{R}} \ln \left(\frac{\mu_{R}^{2}}{m_{t}^{2}}\right)\right] \delta\left(s_{4}\right),
\end{aligned}
$$

where coefficients $C_{0}^{(1)}, C_{1}^{(1)}$ (in which we suppress the ij indices) and $r_{1}$ can be found in appendix B of [61]. At the NNLL accuracy the coefficient $R_{1}$ which consists of virtual graph and soft-gluon radiation contributions, contains the NLO matching term $\operatorname{Tr}\left[H^{(0)} S^{(1)}+H^{(1)} S^{(0)}\right]$. The expression of the NLO matching term is that of the soft plus virtual $(S+V)$ contributions in eq. (6.19) of ref. [18] for the $g g$ channel, and that of the contributions in eq. (4.7) of ref. [20] for the $q \bar{q}$ channel. The Coulomb interactions, due to gluon exchange between the final-state heavy quarks, are included at 1-loop level.

The two-loop corrections at the NNLL accuracy can be written as

$$
\begin{aligned}
\omega_{i j}^{(2)}\left(s_{4}, s, t_{1}, u_{1}\right) & =C_{3}^{(2)}\left[\frac{\ln ^{3}\left(s_{4} / m_{t}^{2}\right)}{s_{4}}\right]_{+}+\left[C_{2}^{(2)}+C_{2, \mu_{F}}^{(2)} \ln \left(\frac{\mu_{F}^{2}}{m_{t}^{2}}\right)\right]\left[\frac{\ln ^{2}\left(s_{4} / m_{t}^{2}\right)}{s_{4}}\right]_{+} \\
+ & {\left[C_{1}^{(2)}+C_{1, \mu_{F}}^{(2)} \ln \left(\frac{\mu_{F}^{2}}{m_{t}^{2}}\right)+C_{1, \mu_{R}}^{(2)} \ln \left(\frac{\mu_{R}^{2}}{m_{t}^{2}}\right)+\bar{C}_{1, \mu_{F}}^{(2)} \ln ^{2}\left(\frac{\mu_{F}^{2}}{m_{t}^{2}}\right)\right]\left[\frac{\ln \left(s_{4} / m_{t}^{2}\right)}{s_{4}}\right]_{+} } \\
+ & {\left[C_{0}^{(2)}+C_{0, \mu_{F}}^{(2)} \ln \left(\frac{\mu_{F}^{2}}{m_{t}^{2}}\right)+C_{0, \mu_{R}}^{(2)} \ln \left(\frac{\mu_{R}^{2}}{m_{t}^{2}}\right)\right.} \\
& \left.+\bar{C}_{0, \mu_{F}}^{(2)} \ln ^{2}\left(\frac{\mu_{F}^{2}}{m_{t}^{2}}\right)+\bar{C}_{0, \mu_{F}, \mu_{R}}^{(2)} \ln \left(\frac{\mu_{F}^{2}}{m_{t}^{2}}\right) \ln \left(\frac{\mu_{R}^{2}}{m_{t}^{2}}\right)\right]\left[\frac{1}{s_{4}}\right]_{+} \\
+ & {\left[R_{2}+r_{2, \mu_{R}} \ln \left(\frac{\mu_{R}^{2}}{m_{t}^{2}}\right)+r_{2, \mu_{F}} \ln \left(\frac{\mu_{F}^{2}}{m_{t}^{2}}\right)+r_{2, \mu_{F}, \mu_{R}} \ln \left(\frac{\mu_{F}^{2}}{m_{t}^{2}}\right) \ln \left(\frac{\mu_{R}^{2}}{m_{t}^{2}}\right)\right.} \\
+ & \left.\bar{r}_{2, \mu_{R}} \ln ^{2}\left(\frac{\mu_{R}^{2}}{m_{t}^{2}}\right)+\bar{r}_{2, \mu_{F}} \ln ^{2}\left(\frac{\mu_{F}^{2}}{m_{t}^{2}}\right)\right] \delta\left(s_{4}\right)
\end{aligned}
$$



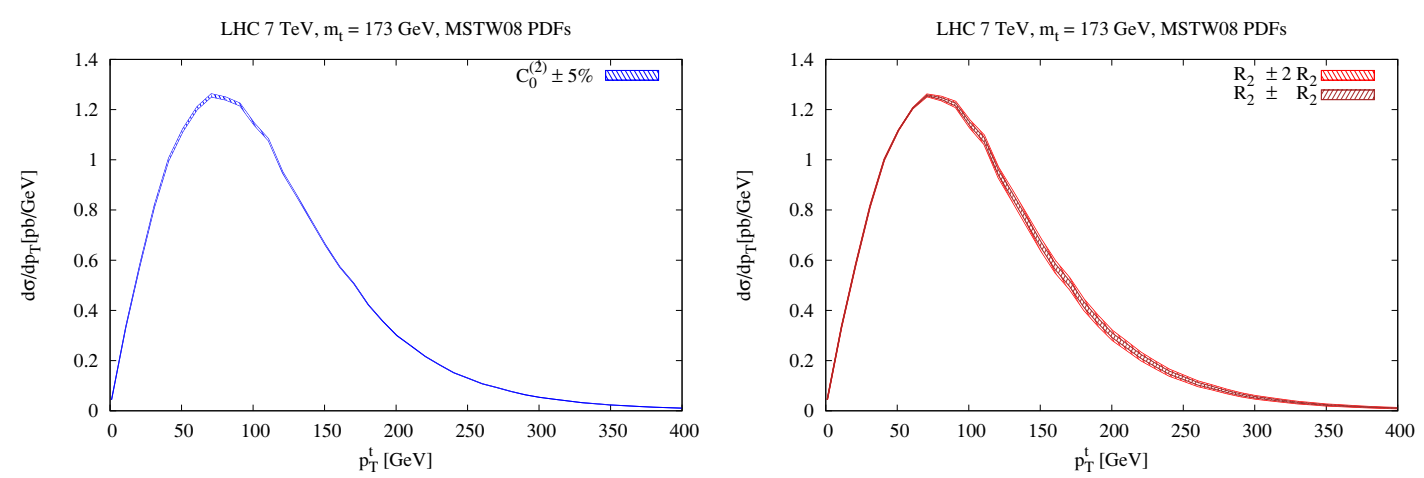

Figure 2. Left: top-quark $p_{T}$ spectrum in which the coefficient $C_{0}^{(2)}$ is varied within its $5 \%$ while $R_{2}$ is kept fixed. Right: here the coefficient $R_{2}$ is varied by adding and subtracting $2 R_{2}$ while $C_{0}^{(2)}$ is kept fixed.

where again, explicit expressions for the coefficients $C_{0, \mu_{F}}^{(2)}, C_{1}^{(2)}, \ldots$ can be found in appendix $\mathrm{B}$ of [61]. In the current implementation the scale-independent coefficient $C_{0}^{(2)}$ contains the contribution from the soft anomalous dimension $\Gamma_{S}^{(2)}$ given in $[83,84]$ plus process-independent terms $[63,82]$ which are universal in the $q \bar{q}$ and $g g$ channels respectively. These contributions are formally at the next-to-next-to-next-leading logarithmic (NNNLL) accuracy. At this level of accuracy, $C_{0}^{(2)}$ is therefore not exact due to incomplete separation of the individual color structures. From a comparison with the analogous approximation for the inclusive cross section we expect only small numerical deviations for the exact result. In the scale-independent coefficient $R_{2}$ we only include NNNLL subleading terms coming from moment to momentum space inversion [60]. The full knowledge of $R_{2}$ requires matching conditions at NNLO which include explicit analytical expressions for $H^{(2)}$ and $S^{(2)}$. In the given kinematics (1PI), these are currently not available for $H^{(2)}$ and $S^{(2)}$ and those terms are thus set to zero. The current uncertainty on $R_{2}$ is therefore the dominant source.

To give an estimate of the dependence of the $p_{T}$ spectrum on the coefficients $C_{0}^{(2)}$ and $R_{2}$ that are known only partially in our computation, we generated a set of predictions in which these coefficients are varied. In figure 2 we illustrate these variations separately, where the coefficient $C_{0}^{(2)}$ is varied within $5 \%$ of its magnitude by keeping $R_{2}$ as fixed, and $R_{2}$ is varied by adding and subtracting $R_{2}$ or $2 R_{2}$, and $C_{0}^{(2)}$ is fixed. Variations of $C_{0}^{(2)}$ produce modifications of magnitude and shape of the $p_{T}$ spectrum which are more pronounced in the peak region, where these are approximately $1 \%$ when $R_{2}$ is fixed. Being $R_{2}$ the less known contribution, we allow this coefficient to vary in a much larger interval to be more conservative. Variations of $R_{2}$ within its $5 \%$ when $C_{0}^{(2)}$ is fixed, are found to be negligible. In figure 2 larger variations of $R_{2}$ have an impact over all the $p_{T}$ range beyond the peak, where modifications of the magnitude and shape can be approximately $7-10 \%$ or more at $p_{T} \approx 200 \mathrm{GeV}$ and are larger than those obtained by a simultaneous variation of factorization and renormalization scales. The uncertainties relative to $C_{0}^{(2)}$ and $R_{2}$ are part of the systematic uncertainty associated to approximate calculations of this kind which are based on threshold expansions. Explicit expressions in SCET for $S^{(2)}$ in PIM kinematic, 
in the limit of boosted top quarks, can be found in ref. [77]. Related studies are presented in refs. [29, 30, 77, 85-87].

By setting $\mu_{R}=\mu_{F}=\mu$ one can write the inclusive total partonic cross section in terms of scaling functions $f_{i j}^{(k, l)}$ that are dimensionless and depend only on the variable $\eta=s /\left(4 m_{t}^{2}\right)-1$

$$
\sigma_{i j}\left(s, m_{t}^{2}, \mu^{2}\right)=\frac{\alpha_{s}^{2}(\mu)}{m_{t}^{2}} \sum_{k=0}^{\infty}\left(4 \pi \alpha_{s}(\mu)\right)^{k} \sum_{l=0}^{k} f_{i j}^{(k, l)}(\eta) \ln ^{l}\left(\frac{\mu^{2}}{m_{t}^{2}}\right) .
$$

To reduce the impact of threshold logarithms in the large- $\eta$ regions of the scaling functions we made use of dumping factors as it is done in ref. [19].

\section{Sensitivity of $t \bar{t}$ production to PDF-related aspects of QCD}

This section addresses details of the phenomenological analysis of the uncertainties on the predictions for differential $t \bar{t}$ production cross sections at the LHC. In particular, PDF uncertainties are studied and compared to the current experimental precision of the measurements. The approximate NNLO DiffTop predictions, obtained by using different PDF sets, are confronted to the recent measurements of differential distributions for $t \bar{t}$ production at $\sqrt{S}=7 \mathrm{TeV}$ by the CMS [10] and ATLAS [13, 14] collaborations. The theoretical systematic uncertainties associated to variations of PDFs, $\alpha_{s}\left(M_{Z}\right)$, scale, and $m_{t}$, are investigated individually. In particular, transverse momentum $p_{T}^{t}$ and rapidity $y^{t}$ distributions of the final state top-quark, measured by CMS, and the $p_{T}^{t}$ distribution by ATLAS $^{3}$ are studied. The on-shell pole-mass definition for the top-quark mass is used, and the value of $m_{t}=173 \mathrm{GeV}$ is chosen. The scales are set to $\mu_{F}=\mu_{R}=m_{t}$. The experimental measurements published by CMS and ATLAS are differential distributions that are normalized to the total cross section in bins of $p_{T}^{t}$ and $y^{t}$. This representation of experimental data is motivated by (partial) cancellation of systematic uncertainties.

The correlation between the $p_{T}^{t}$ distribution for $t \bar{t}$ production at the LHC at $\sqrt{S}=$ $7 \mathrm{TeV}$ and the gluon, as a function of $x$ of the gluon $\left(x_{\text {gluon }}\right)$ is illustrated in figure 3. A strong correlation is observed at $x_{\text {gluon }} \geq 0.01$. Here, the $p_{T}^{t}$ distribution is averaged in 4 bins and the correlation cosine $\cos \phi$, as defined in [88], is evaluated for each bin. The predictions using MSTW08 [89] (left) and CT10 [90] (right) PDFs at NNLO are shown here, while those using ABM11 [91], HERAPDF1.5 [92], and NNPDF2.3 [93] have similar behaviors.

The uncertainties associated to the various PDFs are computed by using the prescription given by each PDF group. All envelopes represent the $68 \%$ confidence level (CL). For the CT10 case, being the CT10NNLO PDF given at 90\% CL, the asymmetric PDF errors are rescaled by a factor 1.642 . In the ABM11 case, the total uncertainty obtained by using the symmetric formula for the eigenvector sets, represents the $\mathrm{PDF}+\alpha_{s}$ uncertainty at the $68 \%$ CL. The uncertainty of HERAPDF1.5 NNLO is determined by including the experimental (at the $68 \% \mathrm{CL}$ ), model and parametrization uncertainties, which are summed in quadrature to obtain the total error.

\footnotetext{
${ }^{3}$ In the data set we considered, $y^{t}$ is not provided by the ATLAS collaboration.
} 

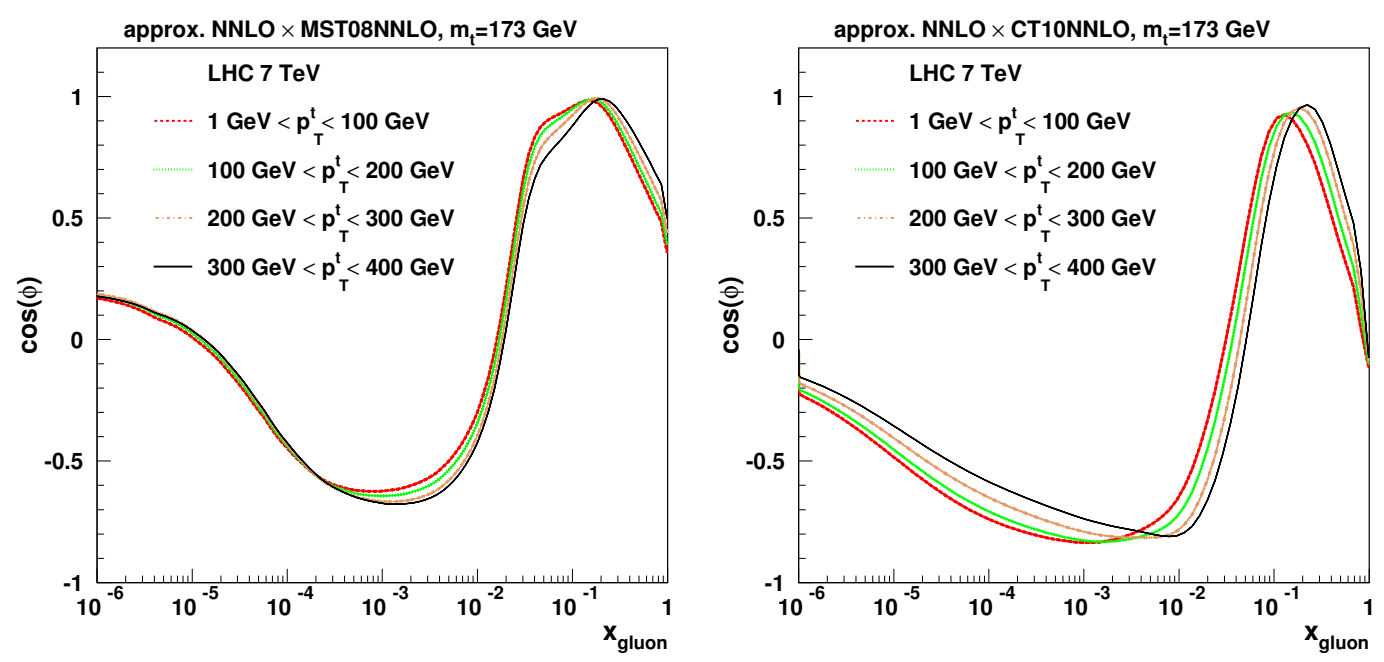

Figure 3. Theoretical correlation cosine as a function of $x_{\text {gluon }}$ for the $p_{T}^{t}$ distribution in $t \bar{t}$ production at the LHC at $\sqrt{S}=7 \mathrm{TeV}$. MSTW08 (left) and CT10 (right) PDFs at NNLO are used. Different lines represent each $p_{T}^{t}$ bin.

The uncertainty associated to the variations of $\alpha_{s}\left(M_{Z}\right)$ is computed by using the central $\alpha_{s}\left(M_{Z}\right)$ values given by each PDF group, and by considering up- and down-variations $\Delta \alpha_{s}\left(M_{Z}\right)= \pm 0.001$. This is more conservative with respect to the $68 \%$ CL variation $\Delta \alpha_{s}\left(M_{Z}\right)=0.0007$, reported in the PDG 2012 [94]. The central values for $\alpha_{s}\left(M_{Z}\right)$ provided by the different PDF groups are: 0.1134, 0.118, 0.1176, 0.1171, 0.118 for ABM11 [91], CT10 [90], HERAPDF1.5 [92], MSTW08 [89], and NNPDF2.3 [93], respectively. The HERAPDF1.5 $\alpha_{s}\left(M_{Z}\right)$ uncertainty is obtained by varying $0.1170 \leq \alpha_{s}\left(M_{Z}\right) \leq 0.1190$, resulting in a larger uncertainty on the cross section, as compared to the other PDF sets. ${ }^{4}$

The uncertainty related to the choice of the scale has been estimated by varying $m_{t} / 2 \leq$ $\mu_{R}=\mu_{F} \leq 2 m_{t}$. As shown in figure 1, where the approximate NNLO prediction and the full NLO calculation obtained by MCFM [39] are compared, the reduction of the scale dependence is substantial. When these theory predictions are compared to the LHC data for the $p_{T}^{t}$ distribution, the shape is also modified when passing from NLO to the approximate NNLO. In the approximate NNLO case, the theoretical description of the measurements is significantly improved.

The uncertainty associated to the pole mass $m_{t}$ has been assessed by considering variations $\Delta m_{t}= \pm 1 \mathrm{GeV}$ around the central value $m_{t}=173 \mathrm{GeV}$.

\subsection{Cross sections of $t \bar{t}$ production at approximate NNLO obtained with dif- ferent PDFs}

In table 1 we summarize the results for the $t \bar{t}$ total inclusive cross section at the LHC at $\sqrt{S}=7 \mathrm{TeV}$ for each PDF set with relative uncertainties.

\footnotetext{
${ }^{4}$ The variation corresponding to the inclusion of members 9-11 of the HERAPDF1.5 $\alpha_{s}$ sets gives an increased uncertainty of approximately $\pm 6.3 \%$.
} 


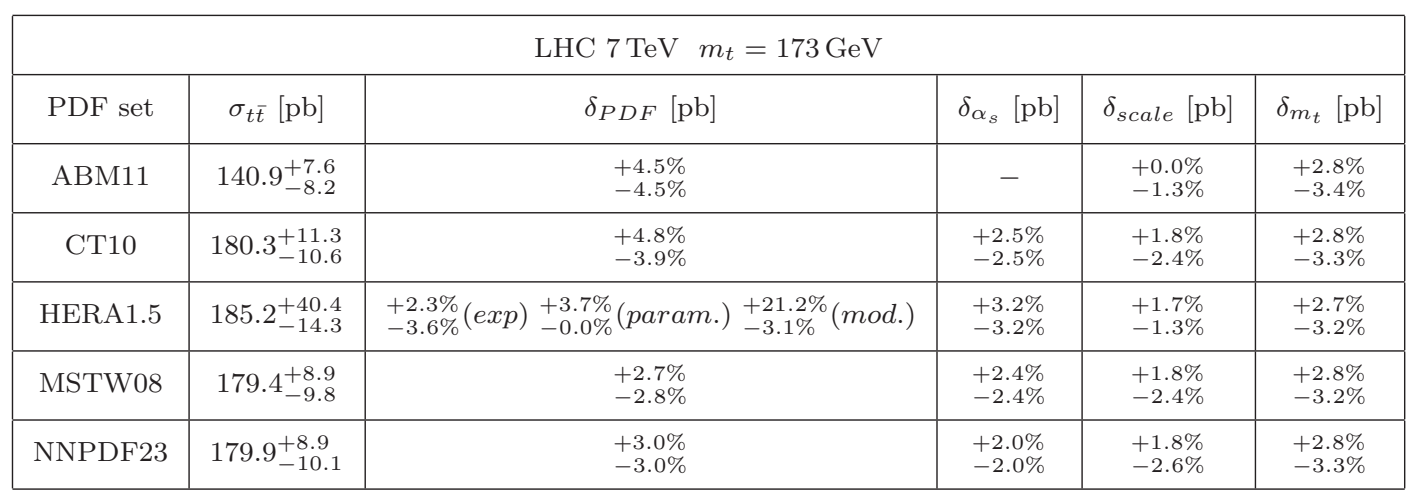

Table 1. Values of the total inclusive $t \bar{t}$ cross section with corresponding uncertainties for PDFs, $\alpha_{s}\left(M_{Z}\right)$, scale dependence and $m_{t}$.

In table 2 we report for comparison the results obtained by using the ToP $++[34]$ code with the same input configuration, where the scale uncertainty is obtained by varying $\mu_{F}$ and $\mu_{R}$ independently. DiffTop predictions are larger than those of ToP ++ by approximately $6-6.5 \%$ in the fixed-order case, and $3-3.5 \%$ in the resummed case. In the fixed-order full NNLO calculation for the inclusive cross section, a simultaneous variation of $\mu_{F}$ and $\mu_{R}$ gives already the full scale variation and all independent variations are included in the envelope $1 / 2 \leq \mu_{F}=\mu_{R} \leq 2$. DifFToP scale uncertainty for simultaneous scale variation is underestimated because of missing contributions in the coefficients $R_{2}$ and $C_{0}^{(2)}$, in particular $R_{2}$, which has impact on the large $p_{T}$-spectrum. Also, missing contributions from the $q g$ channel play a role at higher orders at large $p_{T}$. These missing contributions spoil the agreement in the case of inclusive observables such as the total cross section. However, the local description of differential observables can still be approximated sufficiently well by threshold expansions in regions that are not strongly affected by hard gluon radiation. For example in the region $1 \lesssim p_{T} \lesssim 250-300 \mathrm{GeV}$, where the bulk of the data is currently given. Exact predictions for the fixed-order NLO differential and total inclusive cross sections are in good agreement with the approximate NLO ones (see for example ref. [95]) where the known one-loop soft and hard functions are included. This is an indication of the fact that the approximate NNLO predictions can be improved once the two-loop soft and hard functions from the full NNLO will be available and included in the calculation. A crude estimate of the systematic uncertainties associated to the missing contributions in functions $R_{2}$ and $C_{0}^{(2)}$ for the total and differential cross sections was given in section 3. A more realistic estimate of such uncertainties (variations can actually be combined) requires a separate analysis on which work is in progress and it will be addressed in a forthcoming paper by the authors. In the following figures these uncertainties are therefore not included.

In figure 4 predictions for the absolute $p_{T}^{t}$ and $y^{t}$ distributions are shown, with all used PDF sets that are compared within the respective total uncertainties. The spread of the central values is mostly due to the fact that the parton luminosities are driven by different gluon PDFs of each group. These differences arise from the different methodologies and inputs (heavy-flavor treatment, values of $\alpha_{s}\left(M_{Z}\right)$, data selection, etc.) adopted by each 


\begin{tabular}{|c|c|c|c|c|}
\hline \multicolumn{5}{|c|}{ ToP++ scale dependence at the LHC $7 \mathrm{TeV} m_{t}=173 \mathrm{GeV}$} \\
\hline PDF set & $\sigma_{t \bar{t}}^{N N L O}[\mathrm{pb}]$ & $\mathrm{LL}$ & $\mathrm{NLL}$ & NNLL \\
\hline ABM11 & $134.0_{-8.3(6.2 \%)}^{+5.1(3.8 \%)}$ & $137.1_{-8.2(6.0 \%)}^{+4.6(3.4 \%)}$ & $138.2_{-4.9(3.6 \%)}^{+3.2(2.4 \%)}$ & $138.1_{-4.4(3.2 \%)}^{+3.5(2.5 \%)}$ \\
\hline CT10 & $169.1_{-10.9(6.4 \%)}^{+6.8(4.0 \%)}$ & $173.0_{-10.6(6.1 \%)}^{+6.1(3.5 \%)}$ & $174.3_{-6.7(3.9 \%)}^{+4.3(2.5 \%)}$ & $174.2_{-6.0(3.4 \%)}^{+4.6(2.7 \%)}$ \\
\hline HERA1.5 & $173.6_{-9.4(5.4 \%)}^{+6.6(3.8 \%)}$ & $177.6_{-9.1(5.1 \%)}^{+5.9(3.3 \%)}$ & $178.9_{-3.1(1.7 \%)}^{+2.2(1.3 \%)}$ & $178.8_{-4.2(2.3 \%)}^{+4.8(2.7 \%)}$ \\
\hline MSTW08 & $168.6_{-10.8(6.4 \%)}^{+6.7(4.0 \%)}$ & $172.5_{-10.5(6.1 \%)}^{+6.0(3.5 \%)}$ & $173.7_{-6.6(3.8 \%)}^{+4.2(2.4 \%)}$ & $173.6_{-5.9(3.4 \%)}^{+4.5(2.6 \%)}$ \\
\hline NNPDF23 & $169.1_{-11.1(6.6 \%)}^{+7.0(4.2 \%)}$ & $173.1_{-11.0(6.3 \%)}^{+6.3(3.7 \%)}$ & $174.4_{-6.8(3.9 \%)}^{+4.3(2.4 \%)}$ & $174.3_{-6.0(3.5 \%)}^{+4.6(2.6 \%)}$ \\
\hline
\end{tabular}

Table 2. TOP ++ (ver 2.0) values for the total inclusive $t \bar{t}$ cross section at full NNLO without and with the inclusion of threshold resummation, with scale dependence uncertainty obtained by using independent variations of $\mu_{F}$ and $\mu_{R}$.
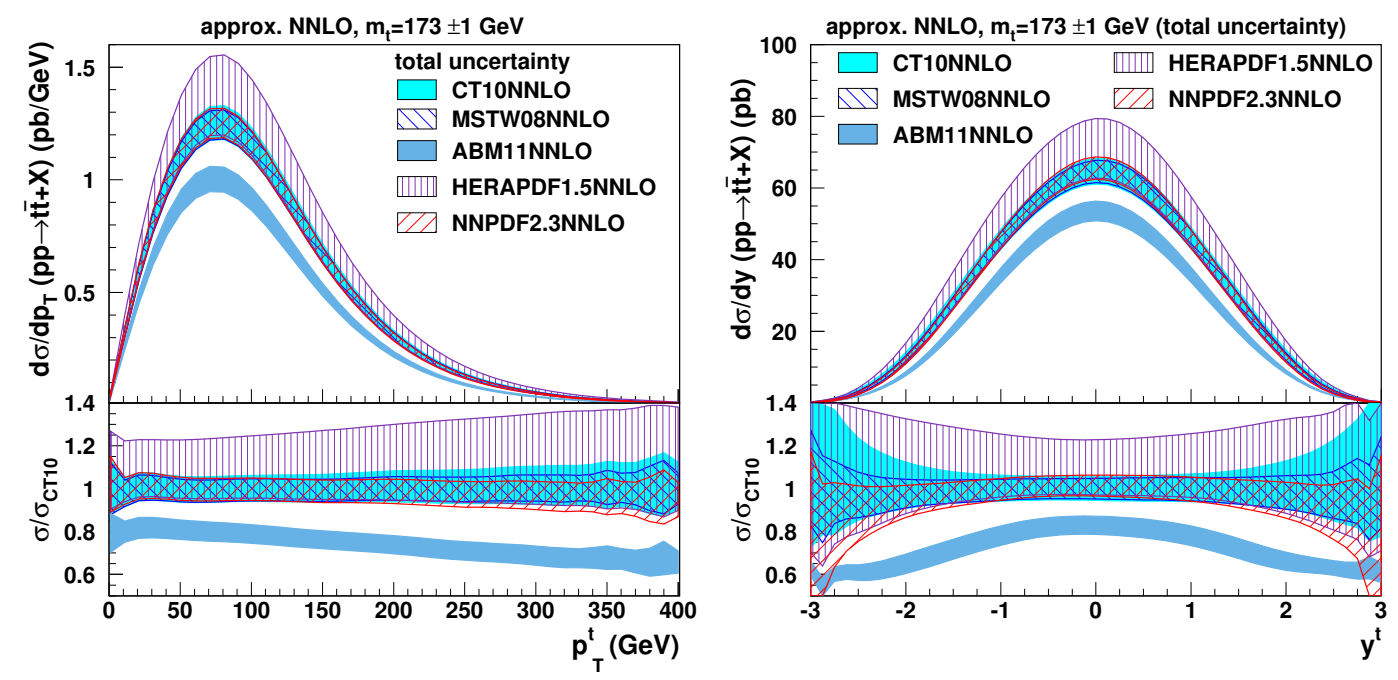

Figure 4. The approximate NNLO predictions for top-quark pair production cross sections at the LHC at $\sqrt{S}=7 \mathrm{TeV}$ as functions of $p_{T}^{t}$ (left) and $y^{t}$ (right). Predictions obtained by using different PDF sets are presented by bands of different hatches. The total uncertainty is obtained by summing the uncertainties due to PDFs, $\alpha_{s}, m_{t}$ and scale variations in quadrature.

PDF fitting group in their QCD analyses. The predictions using ABM11 are found to be generally lower with respect to the other sets, because the different methodology used in the ABM11 analysis leads to a lower gluon and smaller $\alpha_{s}\left(M_{Z}\right)$ value. A larger value of the predicted cross section using ABM11 can be obtained by choosing a smaller value of the top-quark pole mass within the current uncertainties, see, e.g., [96].

Individual contributions to the total uncertainty on the prediction, arising from uncertainties of PDFs and variation of $\alpha_{s}\left(M_{Z}\right)$, scales, and $m_{t}$, are studied for each used PDF set separately. The results for the CT10NNLO PDFs are shown in figure 5, while similar results obtained by using all the other PDF sets can be found in the appendix. The total uncertainty on the theory prediction for the total and absolute differential $t \bar{t}$ cross section is dominated by the PDF errors. At large $p_{T}^{t}$ and $\left|y^{t}\right|$, where regions of larger values of $x$ are 

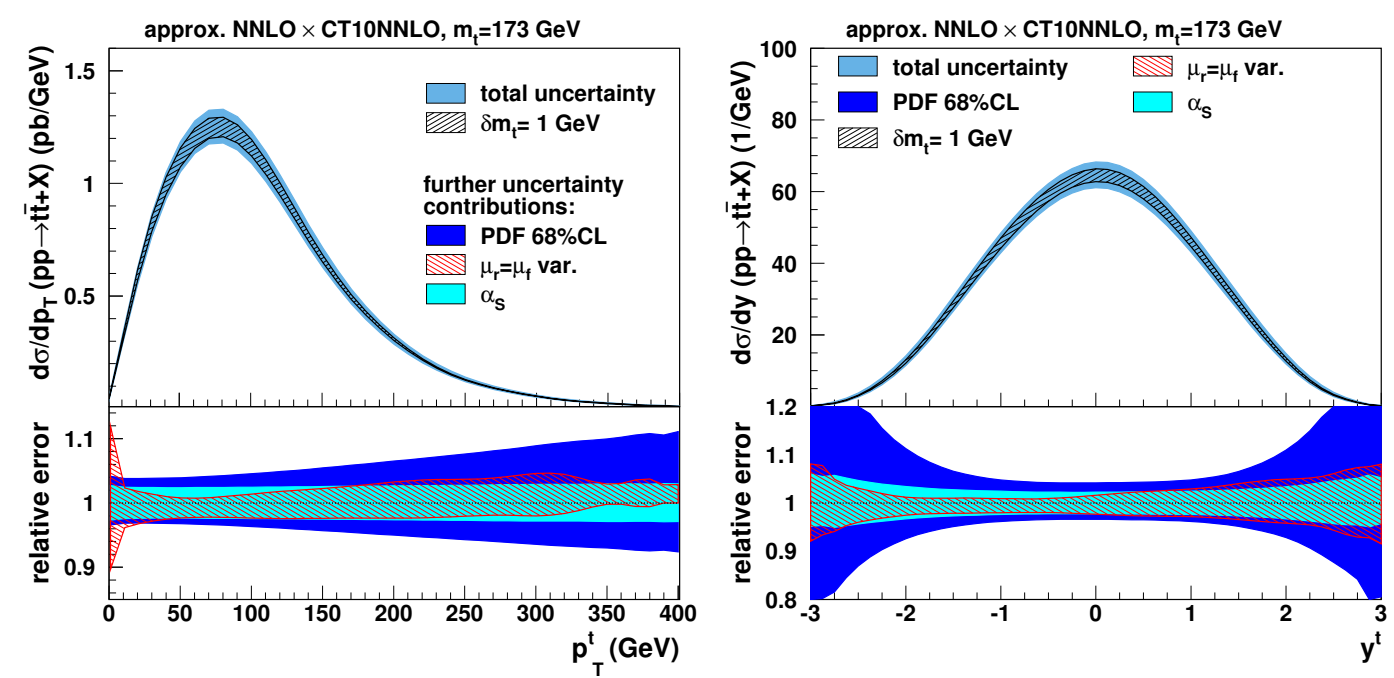

Figure 5. The approximate NNLO predictions for $t \bar{t}$ production cross sections at the LHC at $\sqrt{S}=7 \mathrm{TeV}$ as functions of $p_{T}^{t}$ (left) and $y^{t}$ (right) obtained by using CT10NNLO. The individual contributions of the uncertainties due to the PDFs (68\% CL), $\alpha_{s}\left(M_{Z}\right)$, scale, and $m_{t}$ variations, are shown separately by bands of different shades.

probed, the PDF uncertainties increase since the gluon distribution in this range is poorly constrained at present. In the case of HERAPDF1.5 PDF, in addition to the experimental uncertainty, also parametrization and model uncertainties are estimated. The inclusion of the model uncertainty results in an increase of the cross section by approximately $20 \%$, related to the variation of the $q^{2}$ cut on the used DIS data. In the case of ABM11 PDFs, the $\alpha_{s}$ variation is already included in the quoted PDF error since in the ABM fit $\alpha_{s}$ is simultaneously determined together with the PDFs.

Variations of $m_{t}$ modify the magnitude and shape of the distribution at large- $p_{T}$ and represent the second dominant contribution to the total uncertainty. This reflects the strong sensitivity of the differential distributions to the top-quark mass. The usage of the $\overline{\mathrm{MS}}$ scheme definition for the top-quark mass in the calculation is expected to improve the convergence of perturbation theory and, in turn, to reduce the scale dependence [97]. In some regions of the $p_{T}^{t}$ and $y^{t}$ spectra, the uncertainty associated to variations of $\alpha_{s}\left(M_{Z}\right)$ is sizable. The predictions for the normalized differential cross sections using different PDF sets are shown with their total uncertainties in figure 6 , where these are compared to the recent measurements by the CMS and ATLAS collaborations at $\sqrt{S}=7 \mathrm{TeV}$. The different contributions to the uncertainty of the theory prediction are studied individually and the results for CT10NNLO PDFs are illustrated in figure 7. Similar results obtained by using other PDF sets are shown in the appendix.

When theory predictions are compared to the data, CMS and ATLAS measurements exhibit some differences in the shape of the normalized $p_{T}^{t}$ distribution, in particular in the first and third bin. In general, the first bins of transverse momentum $0 \leq p_{T}^{t} \leq 200 \mathrm{GeV}$ and central bins of rapidity $-1.5 \leq y^{t} \leq 1.5$ have potentially more constraining power, because of smaller experimental uncertainties. At the present stage, even though the CMS 

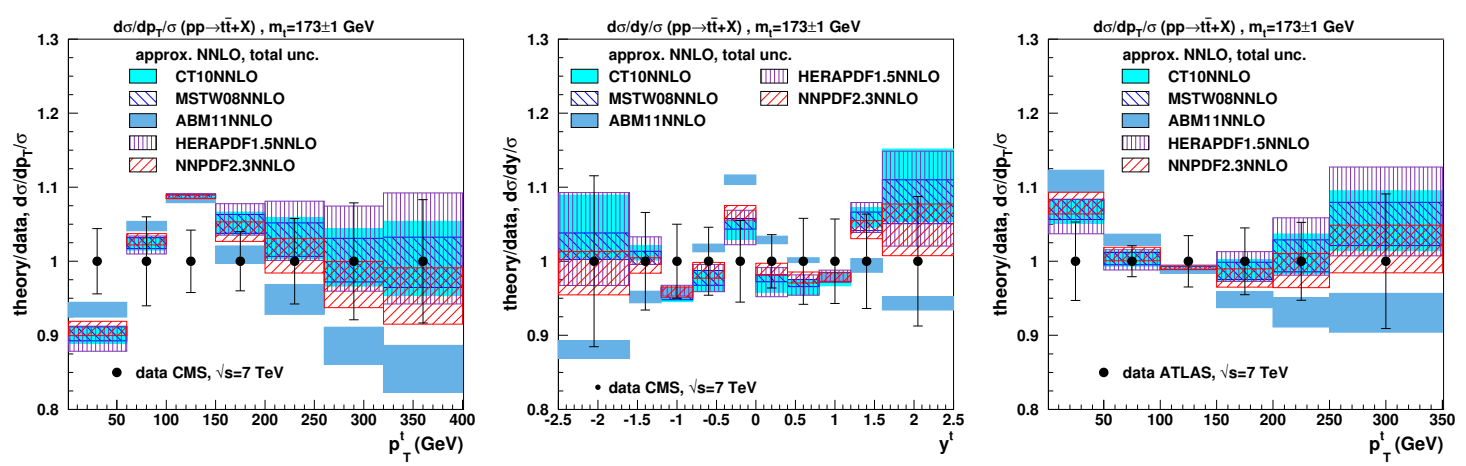

Figure 6. The approximate NNLO predictions for $t \bar{t}$ production at the LHC at $\sqrt{S}=7 \mathrm{TeV}$, shown as functions of $p_{T}^{t}$ and $y^{t}$. The predictions with their total uncertainties, obtained by using different PDF sets (bands of different hatches), are presented as a ratio to the LHC measurements (filled symbols).
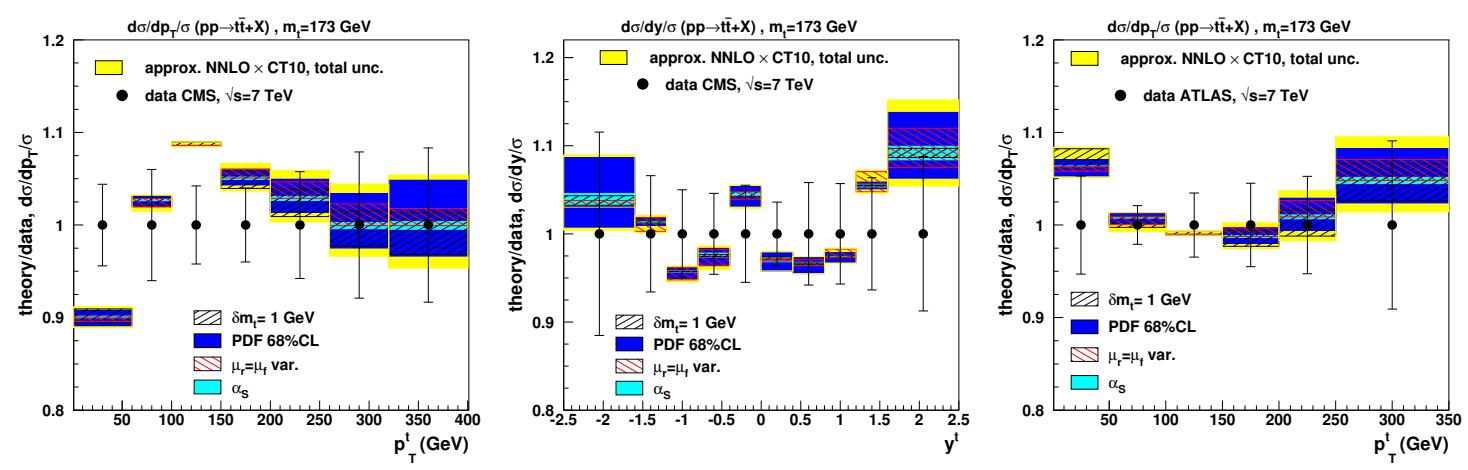

Figure 7. The approximate NNLO prediction for $t \bar{t}$ production at the LHC at $\sqrt{S}=7 \mathrm{TeV}$ obtained by using CT10NNLO PDF as a function of $p_{T}^{t}$ and $y^{t}$. The prediction is presented as a ratio to the LHC measurements (filled symbols). Individual contributions to the theoretical uncertainty are shown by the bands of different shades.

and ATLAS measurements exhibit relatively large uncertainties, these data might have some impact in PDF determination once included in QCD fit analyses.

\subsection{QCD analysis using the $t \bar{t}$ production measurements}

To illustrate the possible impact of the current available measurements of $t \bar{t}$ production on PDF determination, we interfaced the DifFTop code to the HERAFiTTER [51] platform for QCD analyses. Fast theoretical calculations are obtained by using grids generated by the FASTNLO package [48-50]. This allows the user to include measurements of differential $t \bar{t}$ production cross sections into NNLO QCD fits of PDFs. The NNLO PDF fit performed here uses the parton evolution implemented in the QCDNUM [98] code, that is the default parton-evolution package utilized in HERAFITTER. The most important data sets for PDF determination are the combined HERA I measurements [104] of inclusive DIS, which we include in this analysis. Constraints on the $u$ and $d$-quark distributions in the $x$-range not properly covered by the HERA I measurements, are put by the CMS precise measurements of electron [105] and muon [106] charge asymmetry in $W$-boson production at $\sqrt{S}=7 \mathrm{TeV}$. 
The theory predictions for the lepton charge asymmetry are obtained by using the MCFM program at NLO and K-factors are applied in the NNLO fit.

The $t \bar{t}$ measurements included in this analysis are the total inclusive cross sections at the LHC [99-102] at $\sqrt{S}$ of 7 and $8 \mathrm{TeV}$, and CDF [103] Tevatron, as well as the normalized differential cross-sections $[10,13]$ of $t \bar{t}$ production at the LHC at $\sqrt{S}=7 \mathrm{TeV}$ as a function of $p_{T}^{t}$. The procedure for the determination of the PDFs follows the approach used in the QCD fits of the HERA [104] and CMS [106] collaborations. In our PDF analysis, the TR' $[89,107]$ general mass variable flavor number scheme at NNLO is used for the treatment of heavy-quark contributions with input values of the heavy-quark masses given by $m_{c}=1.4 \mathrm{GeV}$ and $m_{b}=4.75 \mathrm{GeV}$, while the choice of the QCD scales is $\mu_{R}=\mu_{F}=Q$. The strong coupling constant is set to $\alpha_{s}\left(m_{Z}\right)=0.1176$ and the $Q^{2}$ range of the HERA data is restricted to the range $Q^{2} \geq Q_{\min }^{2}=3.5 \mathrm{GeV}^{2}$. The following independent combination of parton distributions is chosen at the QCD evolution initial scale $Q_{0}^{2}=1.9 \mathrm{GeV}^{2}$ in the fitting procedure: $x u_{\mathrm{v}}(x), x d_{\mathrm{v}}(x), x g(x)$ and $x \overline{\mathrm{U}}(x), x \overline{\mathrm{D}}(x)$, where $x \overline{\mathrm{U}}(x)=x \bar{u}(x)$ and $x \overline{\mathrm{D}}(x)=x \bar{d}(x)+x \bar{s}(x)$. At the scale $Q_{0}$, the parton distributions are represented by

$$
\begin{aligned}
x u_{\mathrm{v}}(x) & =A_{u_{\mathrm{v}}} x^{B_{u_{\mathrm{v}}}}(1-x)^{C_{u_{\mathrm{v}}}}\left(1+D_{u_{\mathrm{v}}} x+E_{u_{\mathrm{v}}} x^{2}\right), \\
x d_{\mathrm{v}}(x) & =A_{d_{\mathrm{v}}} x^{B_{d_{\mathrm{v}}}}(1-x)^{C_{d_{\mathrm{v}}}} \\
x \overline{\mathrm{U}}(x) & =A_{\overline{\mathrm{U}}} x^{B_{\overline{\mathrm{U}}}}(1-x)^{C_{\overline{\mathrm{U}}}} \\
x \overline{\mathrm{D}}(x) & =A_{\overline{\mathrm{D}}} x^{B_{\overline{\mathrm{D}}}}(1-x)^{C_{\overline{\mathrm{D}}}} \\
x g(x) & =A_{g} x^{B_{g}}(1-x)^{C_{g}}+A_{g}^{\prime} x^{B_{g}^{\prime}}(1-x)^{C_{g}^{\prime}} .
\end{aligned}
$$

The normalization parameters $A_{u_{\mathrm{v}}}, A_{d_{\mathrm{v}}}, A_{g}$ are determined by the QCD sum rules, the $B$ parameter is responsible for small- $x$ behavior of the PDFs, and the parameter $C$ describes the shape of the distribution as $x \rightarrow 1$. A flexible form for the gluon distribution is adopted with the choice of $C_{g}^{\prime}=25$ motivated by the approach of the MSTW group [89, 107]. The analysis is performed by fitting 14 free parameters in eqs. (4.1)-(4.5). Additional constraints $B_{\overline{\mathrm{U}}}=B_{\overline{\mathrm{D}}}$ and $A_{\overline{\mathrm{U}}}=A_{\overline{\mathrm{D}}}\left(1-f_{s}\right)$ are imposed by $f_{s}$ that is the strangeness fraction defined as $f_{s}=\bar{s} /(\bar{d}+\bar{s})$, which is fixed to $f_{s}=0.31 \pm 0.08$ as in the analysis of ref. [89].

A comparison of the PDFs resulting from the fit obtained by using only the HERA DIS data and that obtained by employing the HERA DIS in addition to the CMS lepton charge asymmetry measurements, shows effects on the central value of the light-quark distributions and on the reduction of the uncertainties which are similar to the findings reported by the CMS collaboration in a recent QCD analysis [106] at NLO. A slight reduction of the uncertainty of the gluon distribution in the HERA DIS + CMS lepton asymmetry fit with respect to the fit including the HERA DIS only, is ascribed to the improved constraints on the light-quark distributions through the sum rules.

The inclusion the $t \bar{t}$ measurements in the current NNLO PDF fit leads to a change of the shape of the gluon distribution and a moderate improvement of its uncertainty at large $x$. This is observed in particular at high scales, as illustrated in figure 8. By increasing the scale, the quantitative reduction of the uncertainty of the gluon distribution remains similar, but it sets in at lower values of $x$. A similar effect is observed, although 

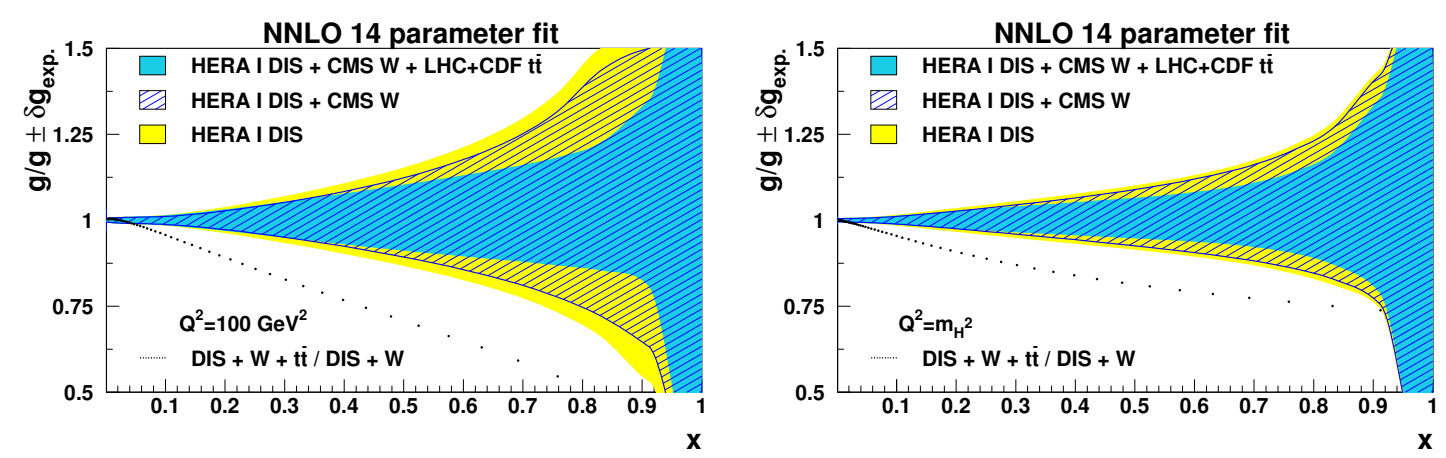

Figure 8. Uncertainties of the gluon distribution as a function of $x$, as obtained in our NNLO fit by using: inclusive DIS measurements only (light shaded band), DIS and $W$ lepton charge asymmetry data (hatched band), and DIS, lepton charge asymmetry and the $t \bar{t}$ production measurements (dark shaded band), shown at the scales of $Q^{2}=100 \mathrm{GeV}^{2}$ (left) and $Q^{2}=m_{H}^{2}$ (right). The ratio of $g(x)$ obtained in the fit including $t \bar{t}$ data to that obtained by using DIS and lepton charge asymmetry, is represented by a dotted line.

less pronounced, when only the total or only the differential $t \bar{t}$ cross section measurements are included in the fit. The results of our full PDF fit, demonstrating a moderate improvement of the uncertainty on the gluon distribution, confirm the observation reported in the reweighting analysis [46] which uses only the total $t \bar{t}$ cross sections.

The analysis presented here uses the normalized differential cross sections for $t \bar{t}$ production. The use of normalized data leads to partial cancellation or reduction of the experimental uncertainties. However, a significant amount of information is lost by normalizing the data, in particular, in connection to uncertainty correlations, which are currently not provided by the experimental collaborations. Measurements of absolute differential cross sections supplied by full information about correlation of experimental uncertainties are of crucial importance to fully exploit the potential of the $t \bar{t}$ production to constrain the gluon distribution. Furthermore, the dependence of the experimentally measured differential $t \bar{t}$ cross section on the assumptions on the top-quark mass used in the Monte Carlo simulations used for efficiency calculation, is necessary. In the future, a reduction of the statistic and systematic uncertainties in the high-energy run of the LHC will be of clear advantage. A simultaneous determination of the gluon distribution and the top-quark mass is of particular interest.

\section{Summary and conclusions}

In this paper, we present results for the differential cross section of $t \bar{t}$ production at approximate NNLO that are of interest for phenomenological studies at hadron colliders. This calculation is implemented into the flexible computer code DifFToP, which is a useful tool for precision studies in QCD and phenomenological applications of the $t \bar{t}$ differential cross section.

In particular, details of the predicted distributions of top-quark transverse momentum and rapidity, generated by using different PDF sets are studied and compared to the 
recent measurements by the CMS and ATLAS collaborations. Individual uncertainties due to variations of PDFs, scale, $\alpha_{s}$ and $m_{t}$, are analyzed. The DiffTop code has been interfaced to FASTNLO for fast evaluations of the theory predictions, and it has been included into the HERAFiTTER framework for QCD analysis to determine the PDFs of the proton. For the first time, it is possible to include measurements of the differential cross sections of $t \bar{t}$ production at the LHC into a full PDF fit. We studied the impact of the recent measurements of the inclusive and differential $t \bar{t}$ production cross sections in a PDF fit at NNLO together with the HERA I inclusive DIS data and the CMS measurements of the lepton charge asymmetries in $W$ boson production. Given the current experimental precision of $t \bar{t}$ measurements, a moderate improvement of the uncertainty on the gluon distribution at high $x$ is observed once the total and differential $t \bar{t}$ cross sections are included in the QCD analysis. Measurements of the $p_{T}^{t}$ and $y^{t}$ differential cross sections of $t \bar{t}$ production in hadron collisions are going to play an important role in constraining the gluon PDF at large- $x$ as the LHC will reach higher experimental precision in the forthcoming run II. Given the correlations between the strong coupling $\alpha_{s}\left(M_{Z}\right)$, top-quark mass and gluon PDF, these measurements could be used to constrain the large- $x$ gluon distribution and the top-quark mass simultaneously. In particular, investigations of absolute differential cross sections will bring complementary information related to the magnitude and other details of the distributions, which will be crucial to improve the constraining power of the experimental data.

\section{Acknowledgments}

We would like to thank Ben Pecjak for providing mathematica files with expressions to cross-check part of the results on the matching conditions. We would also like to thank Nick Kidonakis, Maria Aldaya, Ringaile Placakyte and Aleko Khukhunaishvili for useful discussions. We are grateful to Daniel Britzger (FAstNLO Collaboration) for assistance in the implementation of DiffTop in FASTNLO, and for providing us with the corresponding tables. This work is supported in part by the "Initiative and Networking Fund of the Helmholtz Association (HGF) under the contract S0-072", by Deutsche Forschungsgemeinschaft in Sonderforschungsbereich/Transregio 9, by the European Commission through contract PITN-GA-2010-264564 (LHCPhenoNet), and it was realized within the scope of the PROSA collaboration.

Note added. During the review process of this paper, very preliminary results for the exact calculation for the differential cross sections for $t \bar{t}$ production at NNLO in QCD were presented at the TOP2014 conference in Cannes, France. Details can be found at [108]. 


\section{A Comparison with other PDF sets}
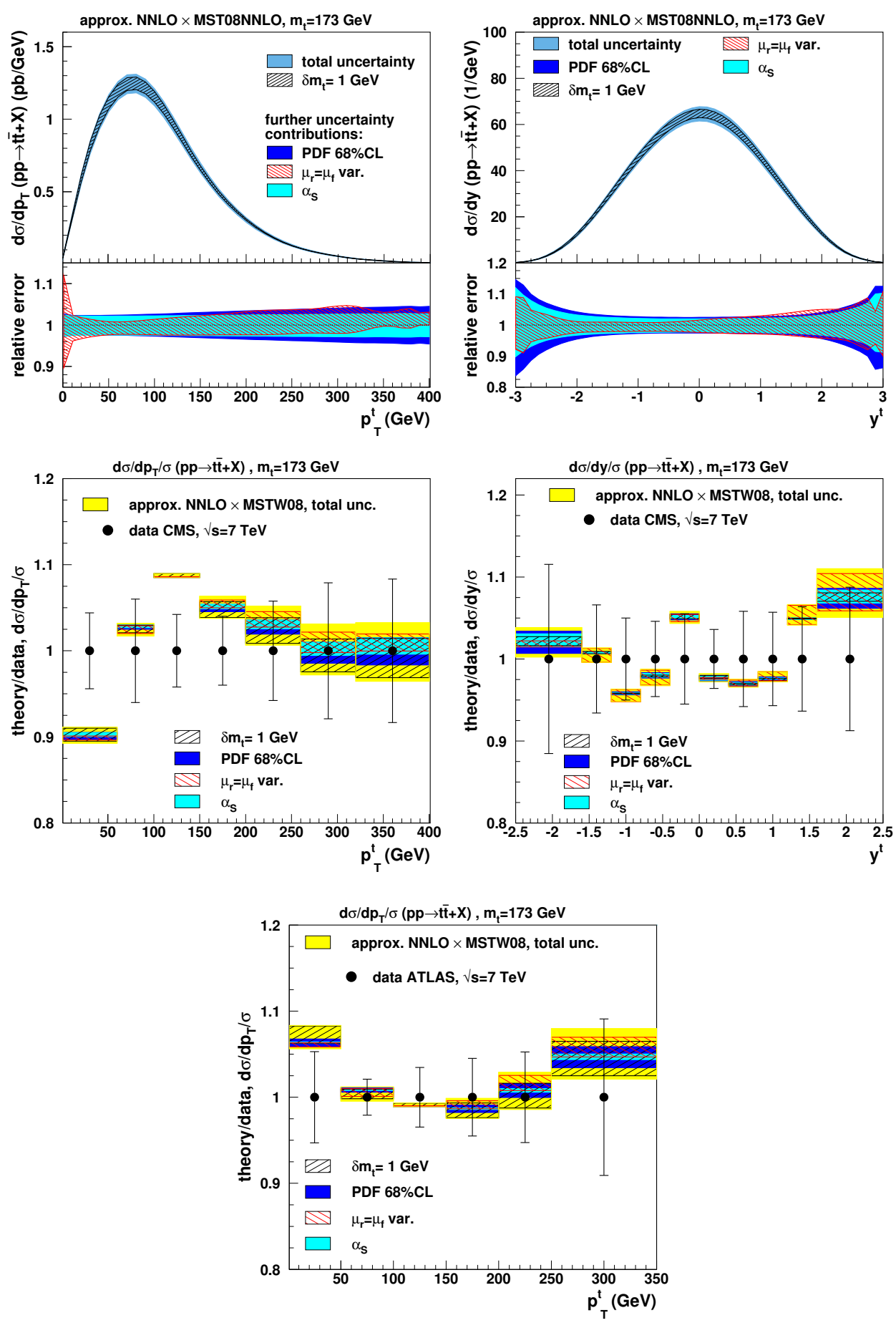

Figure 9. Upper inset: approximate NNLO predictions and the relative uncertainty for $t \bar{t}$ production cross sections as a function of $p_{T}^{t}$ and $y^{t}$ obtained by using MSTW08NNLO. Individual contributions of uncertainties due to PDF $(68 \% \mathrm{CL}), \alpha_{s}\left(M_{Z}\right)$, scale and $m_{t}$ variations are shown by bands of different shades. Lower inset: ratio of theory over data (light shaded band) for $p_{T}^{t}$ and $y^{t}$ as compared to the LHC measurements (filled circles). 

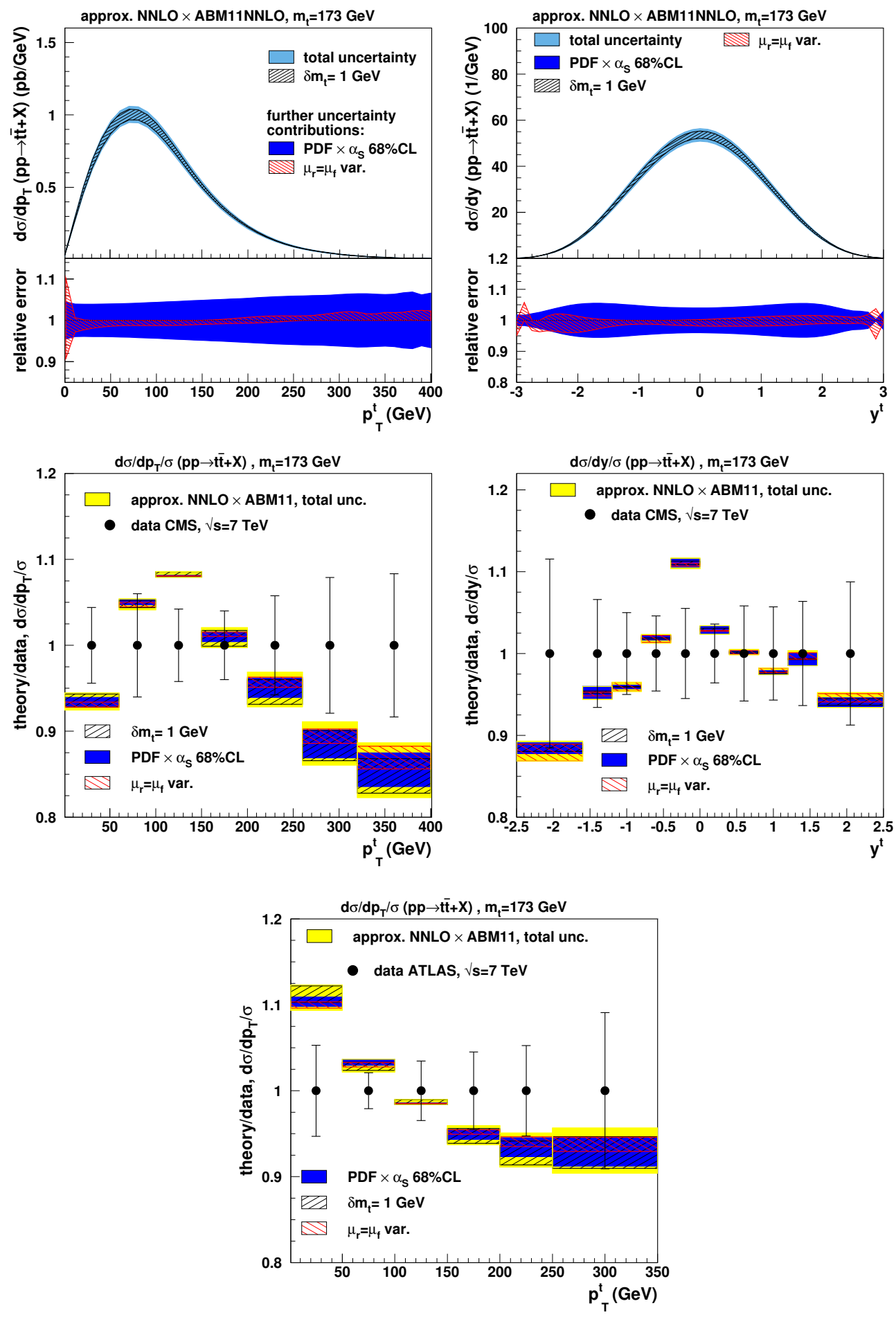

Figure 10. Same as in figure 9 using ABM11 PDFs. Here, the uncertainty on $\alpha_{s}\left(M_{Z}\right)$ is included into the PDF uncertainty, see description in the text. 

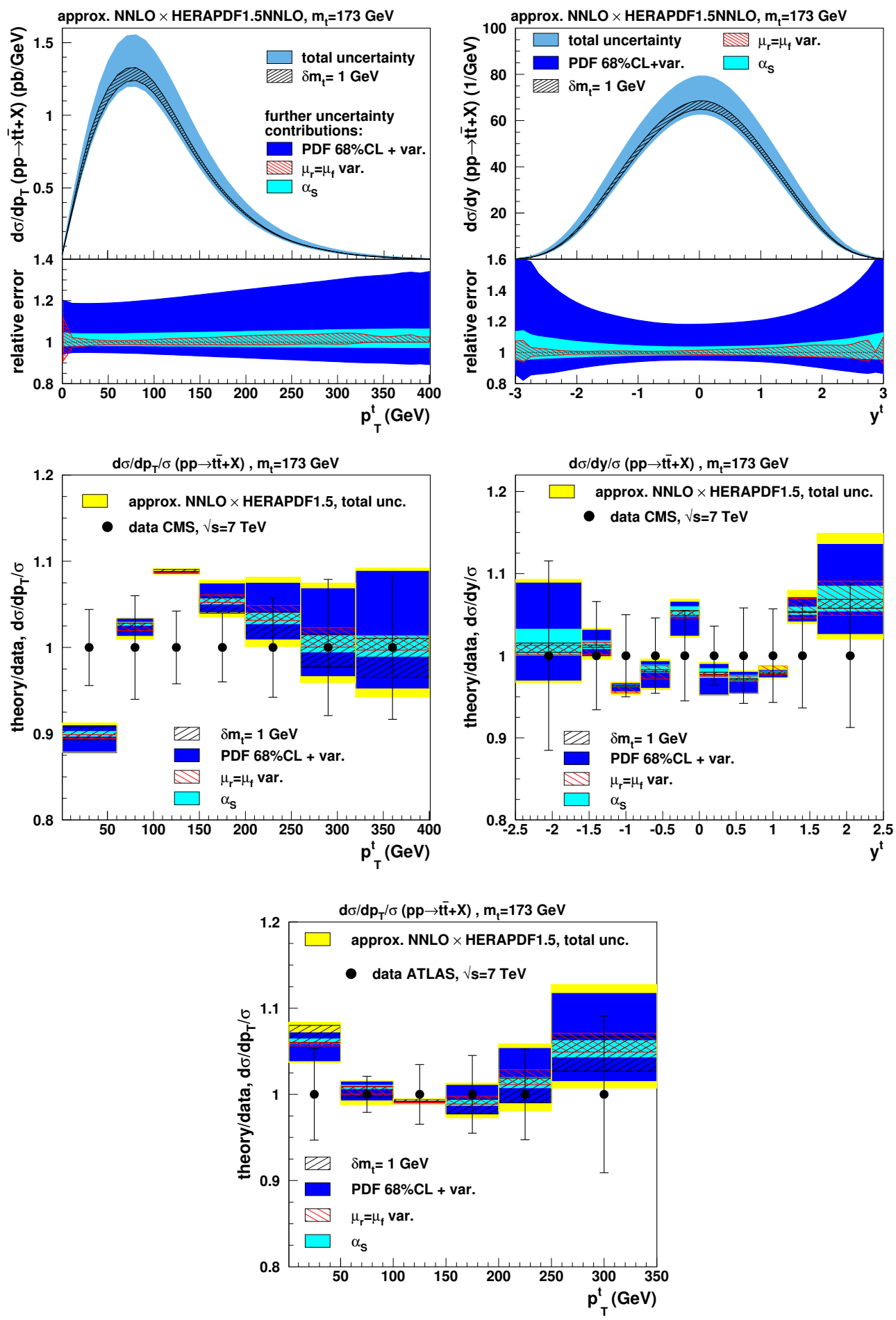

Figure 11. Same as in figure 9 using HERAPDF1.5 NNLO. The experimental uncertainties are given at $68 \%$ CL. In addition, model and parametrization uncertainties are considered. 

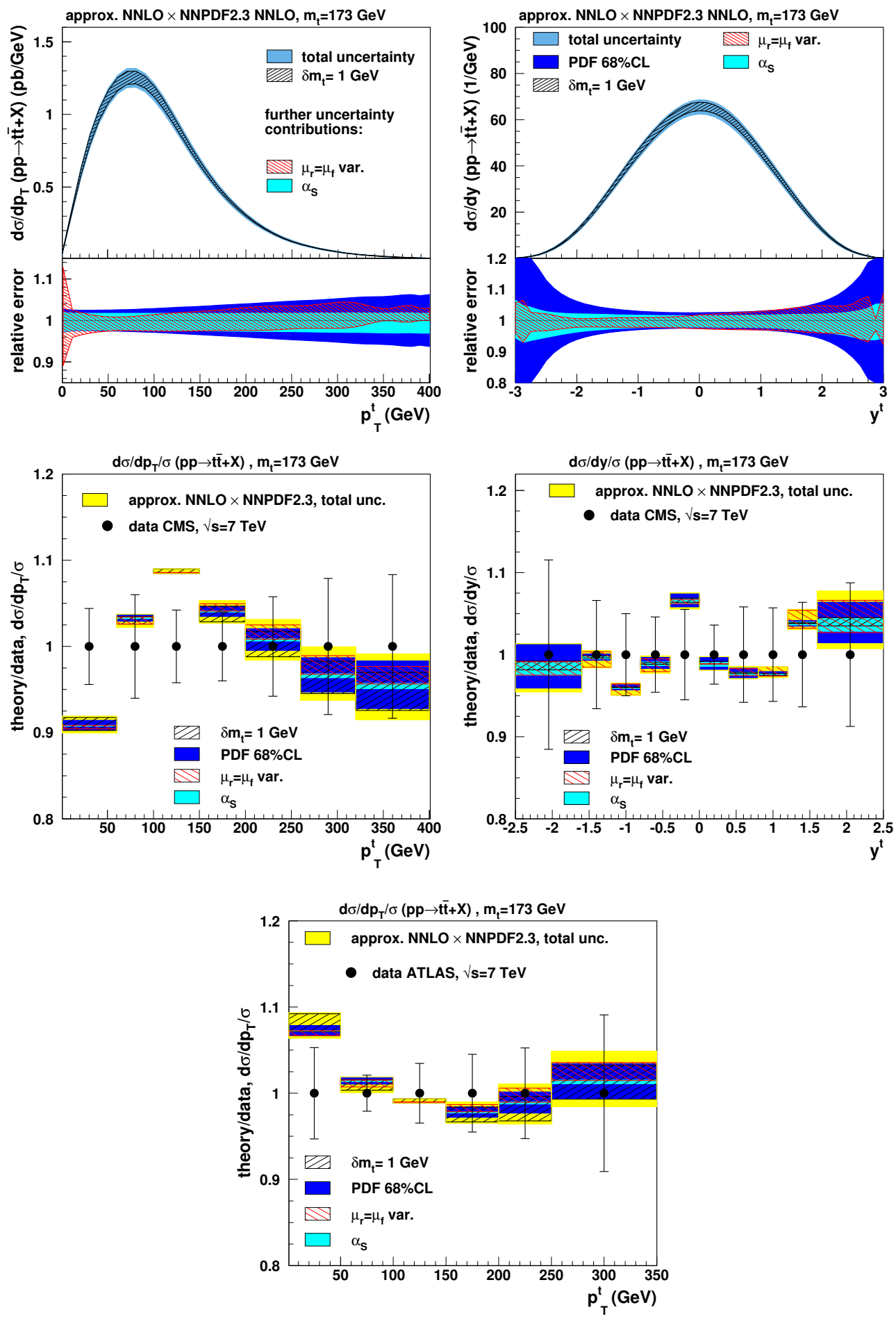

Figure 12. Same as in figure 9 using NNPDF2.3 PDFs. 
Open Access. This article is distributed under the terms of the Creative Commons Attribution License (CC-BY 4.0), which permits any use, distribution and reproduction in any medium, provided the original author(s) and source are credited.

\section{References}

[1] ATLAS collaboration, Observation of a new particle in the search for the standard model Higgs boson with the ATLAS detector at the LHC, Phys. Lett. B 716 (2012) 1 [arXiv: 1207.7214] [INSPIRE].

[2] CMS collaboration, Observation of a new boson at a mass of $125 \mathrm{GeV}$ with the CMS experiment at the LHC, Phys. Lett. B 716 (2012) 30 [arXiv:1207.7235] [INSPIRE].

[3] S. Alekhin, A. Djouadi and S. Moch, The top quark and Higgs boson masses and the stability of the electroweak vacuum, Phys. Lett. B 716 (2012) 214 [arXiv:1207.0980] [INSPIRE].

[4] F. Bezrukov, M.Y. Kalmykov, B.A. Kniehl and M. Shaposhnikov, Higgs boson mass and new physics, JHEP 10 (2012) 140 [arXiv:1205.2893] [INSPIRE].

[5] ATLAS, CDF, CMS, D0 collaboration, First combination of Tevatron and LHC measurements of the top-quark mass, arXiv:1403.4427 [INSPIRE].

[6] S. Moch et al., High precision fundamental constants at the TeV scale, arXiv:1405.4781 [INSPIRE].

[7] S. Alioli et al., A new observable to measure the top-quark mass at hadron colliders, Eur. Phys. J. C 73 (2013) 2438 [arXiv:1303.6415] [InSPIRE].

[8] S. Biswas, K. Melnikov and M. Schulze, Next-to-leading order QCD effects and the top quark mass measurements at the LHC, JHEP 08 (2010) 048 [arXiv: 1006.0910] [INSPIRE].

[9] CMS collaboration, Determination of the top-quark pole mass and strong coupling constant from the $t \bar{t}$ production cross section in pp collisions at $\sqrt{s}=7 \mathrm{TeV}$,

Phys. Lett. B 728 (2014) 496 [Erratum ibid. B 728 (2014) 526-528] [arXiv:1307.1907] [INSPIRE].

[10] CMS collaboration, Measurement of differential top-quark pair production cross sections in pp colisions at $\sqrt{s}=7 \mathrm{TeV}$, Eur. Phys. J. C 73 (2013) 2339 [arXiv:1211.2220] [inSPIRE].

[11] CMS collaboration, Measurement of the t $\bar{t}$ production cross section in the dilepton channel in pp collisions at $\sqrt{s}=8 \mathrm{TeV}$, JHEP 02 (2014) 024 [Erratum ibid. 1402 (2014) 102] [arXiv:1312.7582] [INSPIRE].

[12] ATLAS collaboration, Measurements of top quark pair relative differential cross-sections with ATLAS in pp collisions at $\sqrt{s}=7$ TeV, Eur. Phys. J. C 73 (2013) 2261 [arXiv: 1207.5644] [INSPIRE].

[13] ATLAS collaboration, Measurements of normalized differential cross-sections for $t \bar{t}$ production in pp collisions at $\sqrt{s}=7 \mathrm{TeV}$ using the ATLAS detector, CERN-PH-EP-2014-099 (2014).

[14] ATLAS collaboration, Measurement of top-quark pair differential cross-sections in the $l+$ jets channel in pp collisions at $\sqrt{s}=7 \mathrm{TeV}$ using the ATLAS detector, ATLAS-CONF-2013-099 (2013) [ATLAS-COM-CONF-2013-114].

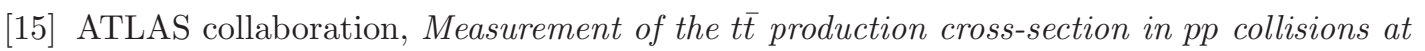
$\sqrt{s}=8 \mathrm{TeV}$ using e $\mu$ events with b-tagged jets, ATLAS-CONF-2013-097 (2013)

[ATLAS-COM-CONF-2013-112]. 
[16] P. Nason, S. Dawson and R.K. Ellis, The total cross-section for the production of heavy quarks in hadronic collisions, Nucl. Phys. B 303 (1988) 607 [INSPIRE].

[17] P. Nason, S. Dawson and R.K. Ellis, The one particle inclusive differential cross-section for heavy quark production in hadronic collisions, Nucl. Phys. B 327 (1989) 49 [Erratum ibid. B 335 (1990) 260] [INSPIRE].

[18] W. Beenakker, H. Kuijf, W.L. van Neerven and J. Smith, QCD corrections to heavy quark production in pp collisions, Phys. Rev. D 40 (1989) 54 [InSPIRE].

[19] R. Meng, G.A. Schuler, J. Smith and W.L. van Neerven, Simple formulae for the order $\alpha^{-} s^{3}$ $Q C D$ corrections to the reaction $p \bar{p} \rightarrow Q \bar{Q} X$, Nucl. Phys. B 339 (1990) 325 [INSPIRE].

[20] W. Beenakker, W.L. van Neerven, R. Meng, G.A. Schuler and J. Smith, QCD corrections to heavy quark production in hadron hadron collisions, Nucl. Phys. B 351 (1991) 507 [INSPIRE].

[21] M.L. Mangano, P. Nason and G. Ridolfi, Heavy quark correlations in hadron collisions at next-to-leading order, Nucl. Phys. B 373 (1992) 295 [InSPIRE].

[22] M. Czakon, P. Fiedler and A. Mitov, Total top-quark pair-production cross section at hadron colliders through $O\left(\alpha_{S}^{4}\right)$, Phys. Rev. Lett. 110 (2013) 252004 [arXiv:1303.6254] [INSPIRE].

[23] M. Czakon and A. Mitov, NNLO corrections to top pair production at hadron colliders: the quark-gluon reaction, JHEP 01 (2013) 080 [arXiv: 1210.6832] [INSPIRE].

[24] M. Czakon and A. Mitov, NNLO corrections to top-pair production at hadron colliders: the all-fermionic scattering channels, JHEP 12 (2012) 054 [arXiv:1207.0236] [INSPIRE].

[25] P. Bärnreuther, M. Czakon and A. Mitov, Percent level precision physics at the Tevatron: first genuine NNLO QCD corrections to $q \bar{q} \rightarrow t \bar{t}+X$, Phys. Rev. Lett. 109 (2012) 132001 [arXiv:1204.5201] [INSPIRE].

[26] M. Czakon, A. Mitov and S. Moch, Heavy-quark production in gluon fusion at two loops in QCD, Nucl. Phys. B 798 (2008) 210 [arXiv:0707.4139] [InSPIRE].

[27] M. Czakon, A. Mitov and S. Moch, Heavy-quark production in massless quark scattering at two loops in QCD, Phys. Lett. B 651 (2007) 147 [arXiv:0705.1975] [InSPIRE].

[28] A. Mitov and S. Moch, The singular behavior of massive QCD amplitudes, JHEP 05 (2007) 001 [hep-ph/0612149] [INSPIRE].

[29] A. Ferroglia, M. Neubert, B.D. Pecjak and L.L. Yang, Two-loop divergences of scattering amplitudes with massive partons, Phys. Rev. Lett. 103 (2009) 201601 [arXiv:0907.4791] [INSPIRE].

[30] A. Ferroglia, M. Neubert, B.D. Pecjak and L.L. Yang, Two-loop divergences of massive scattering amplitudes in non-abelian gauge theories, JHEP 11 (2009) 062 [arXiv:0908.3676] [INSPIRE].

[31] M. Czakon, A novel subtraction scheme for double-real radiation at NNLO, Phys. Lett. B 693 (2010) 259 [arXiv:1005.0274] [InSPIRE].

[32] I. Bierenbaum, M. Czakon and A. Mitov, The singular behavior of one-loop massive QCD amplitudes with one external soft gluon, Nucl. Phys. B 856 (2012) 228 [arXiv:1107.4384] [INSPIRE].

[33] P. Bärnreuther, M. Czakon and P. Fiedler, Virtual amplitudes and threshold behaviour of hadronic top-quark pair-production cross sections, JHEP 02 (2014) 078 [arXiv:1312.6279] [INSPIRE]. 
[34] M. Czakon and A. Mitov, Top++: a program for the calculation of the top-pair cross-section at hadron colliders, Comput. Phys. Commun. 185 (2014) 2930 [arXiv: 1112.5675] [INSPIRE].

[35] M. Aliev et al., HATHOR: HAdronic Top and Heavy quarks crOss section calculatoR, Comput. Phys. Commun. 182 (2011) 1034 [arXiv:1007.1327] [InSPIRE].

[36] A. Denner, S. Dittmaier, S. Kallweit and S. Pozzorini, NLO QCD corrections to WWbb production at hadron colliders, Phys. Rev. Lett. 106 (2011) 052001 [arXiv:1012.3975] [INSPIRE].

[37] G. Bevilacqua, M. Czakon, A. van Hameren, C.G. Papadopoulos and M. Worek, Complete off-shell effects in top quark pair hadroproduction with leptonic decay at next-to-leading order, JHEP 02 (2011) 083 [arXiv: 1012.4230] [INSPIRE].

[38] A. Denner, S. Dittmaier, S. Kallweit and S. Pozzorini, NLO QCD corrections to off-shell top-antitop production with leptonic decays at hadron colliders, JHEP 10 (2012) 110 [arXiv:1207.5018] [INSPIRE].

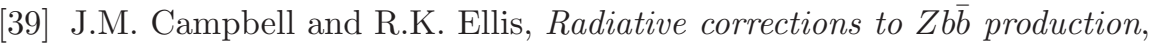
Phys. Rev. D 62 (2000) 114012 [hep-ph/0006304] [inSPIRE].

[40] S. Frixione, P. Nason and B.R. Webber, Matching NLO QCD and parton showers in heavy flavor production, JHEP 08 (2003) 007 [hep-ph/0305252] [INSPIRE].

[41] S. Alioli, K. Hamilton, P. Nason, C. Oleari and E. Re, Jet pair production in POWHEG, JHEP 04 (2011) 081 [arXiv: 1012.3380] [INSPIRE].

[42] J. Alwall et al., MadGraph/MadEvent v4: the new web generation, JHEP 09 (2007) 028 [arXiv: 0706.2334] [INSPIRE].

[43] R. Frederix, S. Frixione, F. Maltoni and T. Stelzer, Automation of next-to-leading order computations in QCD: The FKS subtraction, JHEP 10 (2009) 003 [arXiv:0908.4272] [INSPIRE].

[44] M. Guzzi, K. Lipka and S.-O. Moch, The DiffTop documentation webpage, https://difftop.hepforge.org/.

[45] M. Beneke et al., Inclusive top-pair production phenomenology with TOPIXS, JHEP 07 (2012) 194 [arXiv:1206.2454] [INSPIRE].

[46] M. Czakon, M.L. Mangano, A. Mitov and J. Rojo, Constraints on the gluon PDF from top quark pair production at hadron colliders, JHEP 07 (2013) 167 [arXiv:1303.7215] [INSPIRE].

[47] D. Britzger et al., Generalization of the fastNLO approach to NNLO calculations, talk given at the XXII International Workshop on Deep-Inelastic Scattering and Related Subjects, April 28-May 2, Warsaw, Poland (2014).

[48] FASTNLO collaboration, D. Britzger et al., New features in version 2 of the fastNLO project, arXiv: 1208.3641 [INSPIRE].

[49] FASTNLO collaboration, M. Wobisch et al., Theory-data comparisons for jet measurements in hadron-induced processes, arXiv:1109.1310 [INSPIRE].

[50] T. Kluge, K. Rabbertz and M. Wobisch, FastNLO: fast $p Q C D$ calculations for PDF fits, hep-ph/0609285 [INSPIRE].

[51] HERAFitter web site, http://www.herafitter.org.

[52] T. Carli et al., A posteriori inclusion of parton density functions in NLO QCD final-state calculations at hadron colliders: the APPLGRID project, Eur. Phys. J. C 66 (2010) 503 [arXiv: 0911.2985] [INSPIRE]. 
[53] G.F. Sterman, Summation of large corrections to short distance hadronic cross-sections, Nucl. Phys. B 281 (1987) 310 [InSPIRE].

[54] S. Catani and L. Trentadue, Resummation of the QCD perturbative series for hard processes, Nucl. Phys. B 327 (1989) 323 [InSPIRE].

[55] S. Catani and L. Trentadue, Comment on QCD exponentiation at large $x$, Nucl. Phys. B 353 (1991) 183 [inSPIRE].

[56] N. Kidonakis and G.F. Sterman, Resummation for QCD hard scattering, Nucl. Phys. B 505 (1997) 321 [hep-ph/9705234] [INSPIRE].

[57] E. Laenen, G. Oderda and G.F. Sterman, Resummation of threshold corrections for single particle inclusive cross-sections, Phys. Lett. B 438 (1998) 173 [hep-ph/9806467] [INSPIRE].

[58] R. Bonciani, S. Catani, M.L. Mangano and P. Nason, NLL resummation of the heavy quark hadroproduction cross-section, Nucl. Phys. B 529 (1998) 424 [Erratum ibid. B 803 (2008) 234] [hep-ph/9801375] [INSPIRE].

[59] N. Kidonakis, NNNLO soft-gluon corrections for the top-antitop pair production cross section, Phys. Rev. D 90 (2014) 014006 [arXiv: 1405.7046] [INSPIRE].

[60] N. Kidonakis, High order corrections and subleading logarithms for top quark production, Phys. Rev. D 64 (2001) 014009 [hep-ph/0010002] [INSPIRE].

[61] N. Kidonakis, E. Laenen, S. Moch and R. Vogt, Sudakov resummation and finite order expansions of heavy quark hadroproduction cross-sections, Phys. Rev. D 64 (2001) 114001 [hep-ph/0105041] [INSPIRE].

[62] N. Kidonakis, A Unified approach to NNLO soft and virtual corrections in electroweak, Higgs, QCD and SUSY processes, Int. J. Mod. Phys. A 19 (2004) 1793 [hep-ph/0303186] [INSPIRE].

[63] N. Kidonakis and R. Vogt, Next-to-next-to-leading order soft gluon corrections in top quark hadroproduction, Phys. Rev. D 68 (2003) 114014 [hep-ph/0308222] [INSPIRE].

[64] N. Kidonakis, Next-to-next-to-next-to-leading-order soft-gluon corrections in hard-scattering processes near threshold, Phys. Rev. D 73 (2006) 034001 [hep-ph/0509079] [INSPIRE].

[65] N. Kidonakis and R. Vogt, The theoretical top quark cross section at the Tevatron and the LHC, Phys. Rev. D 78 (2008) 074005 [arXiv:0805.3844] [InSPIRE].

[66] N. Kidonakis, NNLL resummation for s-channel single top quark production, Phys. Rev. D 81 (2010) 054028 [arXiv:1001.5034] [InSPIRE].

[67] N. Kidonakis, Next-to-next-to-leading soft-gluon corrections for the top quark cross section and transverse momentum distribution, Phys. Rev. D 82 (2010) 114030 [arXiv:1009.4935] [INSPIRE].

[68] M. Czakon, A. Mitov and G.F. Sterman, Threshold resummation for top-pair hadroproduction to next-to-next-to-leading log, Phys. Rev. D 80 (2009) 074017 [arXiv:0907.1790] [INSPIRE].

[69] M. Neubert, Heavy quark symmetry, Phys. Rept. 245 (1994) 259 [hep-ph/9306320] [INSPIRE].

[70] C.W. Bauer, S. Fleming, D. Pirjol and I.W. Stewart, An effective field theory for collinear and soft gluons: heavy to light decays, Phys. Rev. D 63 (2001) 114020 [hep-ph/0011336] [INSPIRE].

[71] C.W. Bauer, D. Pirjol and I.W. Stewart, Soft collinear factorization in effective field theory, Phys. Rev. D 65 (2002) 054022 [hep-ph/0109045] [INSPIRE]. 
[72] M. Beneke, A.P. Chapovsky, M. Diehl and T. Feldmann, Soft collinear effective theory and heavy to light currents beyond leading power, Nucl. Phys. B 643 (2002) 431 [hep-ph/0206152] [INSPIRE].

[73] V. Ahrens, A. Ferroglia, M. Neubert, B.D. Pecjak and L.L. Yang, Threshold expansion at order $\alpha_{s}^{4}$ for the $t \bar{t}$ invariant mass distribution at hadron colliders, Phys. Lett. B 687 (2010) 331 [arXiv:0912.3375] [InSPIRE].

[74] V. Ahrens, A. Ferroglia, M. Neubert, B.D. Pecjak and L.L. Yang, Renormalization-group improved predictions for top-quark pair production at hadron colliders, JHEP 09 (2010) 097 [arXiv: 1003.5827] [INSPIRE].

[75] V. Ahrens, A. Ferroglia, M. Neubert, B.D. Pecjak and L.-L. Yang, RG-improved

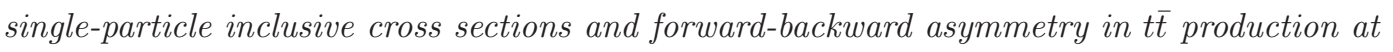
hadron colliders, JHEP 09 (2011) 070 [arXiv:1103.0550] [INSPIRE].

[76] V. Ahrens et al., Precision predictions for the $t+\bar{t}$ production cross section at hadron colliders, Phys. Lett. B 703 (2011) 135 [arXiv:1105.5824] [INSPIRE].

[77] A. Ferroglia et al., The NNLO soft function for the pair invariant mass distribution of boosted top quarks, JHEP 10 (2012) 180 [arXiv:1207.4798] [INSPIRE].

[78] A. Ferroglia, B.D. Pecjak and L.L. Yang, Soft-gluon resummation for boosted top-quark production at hadron colliders, Phys. Rev. D 86 (2012) 034010 [arXiv:1205.3662] [INSPIRE].

[79] A. Ferroglia, S. Marzani, B.D. Pecjak and L.L. Yang, Boosted top production: factorization and resummation for single-particle inclusive distributions, JHEP 01 (2014) 028 [arXiv:1310.3836] [INSPIRE].

[80] N. Kidonakis and B.D. Pecjak, Top-quark production and QCD, Eur. Phys. J. C 72 (2012) 2084 [arXiv:1108.6063] [INSPIRE].

[81] N. Kidonakis and G.F. Sterman, Subleading logarithms in QCD hard scattering, Phys. Lett. B 387 (1996) 867 [INSPIRE].

[82] N. Kidonakis, Top quark production, arXiv:1311.0283 [INSPIRE].

[83] T. Becher and M. Neubert, Infrared singularities of QCD amplitudes with massive partons, Phys. Rev. D 79 (2009) 125004 [Erratum ibid. D 80 (2009) 109901] [arXiv:0904.1021] [INSPIRE].

[84] N. Kidonakis, Two-loop soft anomalous dimensions and NNLL resummation for heavy quark production, Phys. Rev. Lett. 102 (2009) 232003 [arXiv:0903.2561] [INSPIRE].

[85] A. Mitov, G.F. Sterman and I. Sung, The massive soft anomalous dimension matrix at two loops, Phys. Rev. D 79 (2009) 094015 [arXiv: 0903.3241] [INSPIRE].

[86] A. Mitov, G.F. Sterman and I. Sung, Computation of the soft anomalous dimension matrix in coordinate space, Phys. Rev. D 82 (2010) 034020 [arXiv: 1005.4646] [INSPIRE].

[87] M. Czakon and P. Fiedler, The soft function for color octet production at threshold, Nucl. Phys. B 879 (2014) 236 [arXiv:1311.2541] [INSPIRE].

[88] P.M. Nadolsky et al., Implications of CTEQ global analysis for collider observables, Phys. Rev. D 78 (2008) 013004 [arXiv:0802.0007] [inSPIRE].

[89] A.D. Martin, W.J. Stirling, R.S. Thorne and G. Watt, Parton distributions for the LHC, Eur. Phys. J. C 63 (2009) 189 [arXiv:0901.0002] [InSPIRE].

[90] J. Gao et al., CT10 next-to-next-to-leading order global analysis of QCD, Phys. Rev. D 89 (2014) 033009 [arXiv: 1302.6246] [INSPIRE]. 
[91] S. Alekhin, J. Blümlein and S. Moch, Parton distribution functions and benchmark cross sections at NNLO, Phys. Rev. D 86 (2012) 054009 [arXiv:1202.2281] [INSPIRE].

[92] ZEUS, H1 collaboration, A.M. Cooper-Sarkar, PDF fits at HERA, PoS (EPS-HEP2011) 320 [arXiv: 1112.2107] [INSPIRE].

[93] NNPDF collaboration, R.D. Ball et al., Parton distributions with LHC data, Nucl. Phys. B 867 (2013) 244 [arXiv:1207.1303] [INSPIRE].

[94] Particle Data Group collaboration, J. Beringer et al., Review of particle physics, Phys. Rev. D 86 (2012) 010001 [INSPIRE].

[95] M. Guzzi, K. Lipka and S.-O. Moch, Top-quark production at the LHC: differential cross section and phenomenological applications, PoS(DIS 2013) 049 [arXiv:1308.1635] [INSPIRE].

[96] S. Alekhin, J. Blümlein and S. Moch, The ABM parton distributions tuned to LHC data, Phys. Rev. D 89 (2014) 054028 [arXiv:1310.3059] [InSPIRE].

[97] M. Dowling and S.-O. Moch, Differential distributions for top-quark hadro-production with a running mass, Eur. Phys. J. C 74 (2014) 3167 [arXiv:1305.6422] [INSPIRE].

[98] M. Botje, QCDNUM: fast QCD evolution and convolution, Comput. Phys. Commun. 182 (2011) 490 [arXiv: 1005.1481] [INSPIRE].

[99] ATLAS collaboration, Statistical combination of top quark pair production cross-section measurements using dilepton, single-lepton and all-hadronic final states at $\sqrt{s}=7 \mathrm{TeV}$ with the ATLAS detector, ATLAS-CONF-2012-024 (2012).

[100] ATLAS collaboration, Measurement of the top quark pair production cross section in the single-lepton channel with ATLAS in proton-proton collisions at $8 \mathrm{TeV}$ using kinematic fits with b-tagging, ATLAS-CONF-2012-149 (2012).

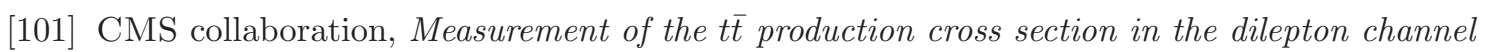
in pp collisions at $\sqrt{s}=7 \mathrm{TeV}$, JHEP 11 (2012) 067 [arXiv:1208.2671] [INSPIRE].

[102] CMS collaboration, Top pair cross section in dileptons, CMS-PAS-TOP-12-007 (2012).

[103] CDF collaboration, Combination of CDF top quark pair production cross section measurements with up to $4.6 \mathrm{fb}^{-1}$, CDF Conference Note 9913 (2009).

[104] H1, ZEUS collaboration, F.D. Aaron et al., Combined measurement and QCD analysis of the inclusive $e^{ \pm} p$ scattering cross sections at HERA, JHEP 01 (2010) 109 [arXiv:0911.0884] [INSPIRE].

[105] CMS collaboration, Measurements of inclusive $W$ and $Z$ cross sections in pp collisions at $\sqrt{s}=7 \mathrm{TeV}, J H E P 01$ (2011) 080 [arXiv:1012.2466] [INSPIRE].

[106] CMS collaboration, Measurement of the muon charge asymmetry in inclusive $p p \rightarrow W+X$ production at $\sqrt{s}=7 \mathrm{TeV}$ and an improved determination of light parton distribution functions, Phys. Rev. D 90 (2014) 032004 [arXiv:1312.6283] [INSPIRE].

[107] R.S. Thorne, A variable-flavor number scheme for NNLO, Phys. Rev. D 73 (2006) 054019 [hep-ph/0601245] [INSPIRE].

[108] M. Czakon and A.D. Mitov, Top pair production, talk given at the Topical workshop on top quark differential distributions (TOP2014), September 26-28, Cannes, France (2014). 